\title{
Review
}

\author{
Giovanna Palermo, Kandammathe Valiyaveedu Sreekanth, Nicolò Maccaferri, \\ Giuseppe Emanuele Lio, Giuseppe Nicoletta, Francesco De Angelis, Michael Hinczewski \\ and Giuseppe Strangi*
}

\section{Hyperbolic dispersion metasurfaces for molecular biosensing}

\author{
https://doi.org/10.1515/nanoph-2020-0466 \\ Received August 9, 2020; accepted September 19, 2020; \\ published online October 7, 2020
}

\begin{abstract}
Sensor technology has become increasingly crucial in medical research and clinical diagnostics to directly detect small numbers of low-molecular-weight biomolecules relevant for lethal diseases. In recent years, various technologies have been developed, a number of them becoming core label-free technologies for detection of cancer biomarkers and viruses. However, to radically improve early disease diagnostics, tracking of disease progression and evaluation of treatments, today's biosensing techniques still require a radical innovation to deliver high sensitivity, specificity, diffusion-limited transport, and accuracy for both nucleic acids and
\end{abstract}

Giovanna Palermo and Kandammathe Valiyaveedu Sreekanth contributed equally to this work.

*Corresponding author: Giuseppe Strangi, Department of Physics, Case Western Reserve University, 10600 Euclid Avenue, Cleveland, $\mathrm{OH}$ 44106, USA; Department of Physics, University of Calabria, Via

P. Bucci, 87036 Rende, CS, Italy; CNR NANOTEC-Istituto di Nanotecnologia, UOS Cosenza, 87036 Rende, CS, Italy,

E-mail: Giuseppe.strangi@case.edu

Giovanna Palermo and Giuseppe Emanuele Lio, Department of Physics, University of Calabria, Via P. Bucci, 87036 Rende, CS, Italy; and CNR NANOTEC-Istituto di Nanotecnologia, UOS Cosenza, 87036 Rende, CS, Italy, E-mail: giovanna.palermo@fis.unical.it (G. Palermo). https://orcid.org/0000-0001-5649-735X (G. Palermo)

Kandammathe Valiyaveedu Sreekanth, Centre for Disruptive Photonic Technologies, The Photonic Institute, Nanyang Technological University, Singapore 637371, Singapore

Nicolò Maccaferri, Department of Physics and Materials Science, University of Luxembourg, L-1511 Luxembourg, Luxembourg. https:// orcid.org/0000-0002-0143-1510

Giuseppe Nicoletta, Department of Physics, University of Calabria, Via P. Bucci, 87036 Rende, CS, Italy

Francesco De Angelis, Istituto Italiano di Tecnologia, I-16163 Genova, Italy

Michael Hinczewski, Department of Physics, Case Western Reserve University, 10600 Euclid Avenue, Cleveland, OH 44106, USA proteins. In this review, we discuss both scientific and technological aspects of hyperbolic dispersion metasurfaces for molecular biosensing. Optical metasurfaces have offered the tantalizing opportunity to engineer wavefronts while its intrinsic nanoscale patterns promote tremendous molecular interactions and selective binding. Hyperbolic dispersion metasurfaces support high- $k$ modes that proved to be extremely sensitive to minute concentrations of ultralow-molecular-weight proteins and nucleic acids.

Keywords: biosensing; hyperbolic dispersion; metamaterials; metasurfaces.

\section{Introduction}

In recent years, a great interest has been spurred by the tremendous technological potential of optically thin nanopatterned surfaces, better known as metasurfaces [112]. Metasurfaces allow wavefront engineering, local phase and amplitude control of light along the surface by using dielectric or plasmonic resonators [13-22]. Plasmonic materials are next-generation nanomaterials with enormous potential to transform health care by providing advanced sensors [23-26], imaging devices [27, 28], and therapies [29-32], as well as to advance energy-relevant materials, such as bio-antennae and light-harvesting systems [33-35]. One promising target for plasmonic sensing technologies is the isolation and detection of circulating tumor cells (CTCs), which have received much attention as novel biomarkers in clinical trials of translational cancer research [36-38]. Because CTCs and other cancer-related biomarkers are present in the blood of many patients with cancer, the detection of CTCs can be considered as a realtime liquid biopsy. It has recently been reported that CTCs can leave primary tumors and enter the circulation at a relatively early stage of tumor growth. Therefore, the detection of CTCs is particularly important to study the mechanism of cancer metastasis [39, 40]. The quantification 
and analysis of CTCs and other cancer-related biomarkers in clinical specimens provide a foundation for the development of future noninvasive liquid biopsy techniques [41-44].

Another class of promising biomarkers is exosomes, which could be useful in early diagnosis of various diseases including cancer, and also for the monitoring of cancer progression through noninvasive or minimally invasive procedures [45-47]. Exosomes are nanoscale (30-150 nm) vesicles released by most cells, including cancer cells, and are found in bodily fluids such as blood, urine, and saliva. Exosomes can transfer their cargo containing proteins, lipids, RNA, and DNA to recipient cells and play a key role in different biological process including intercellular signaling, coagulation, inflammation, and cellular homeostasis $[48,49]$. With respect to exosomes, the main requirements for next-generation sensors are (i) high levels of specificity to isolate exosomes, (ii) the ability to detect low counts of exosomes (100 target cells per $10^{9}$ blood cells), and (iii) the ability to detect in patient whole blood samples. In this direction, a plasmonic-based, label-free, high-throughput exosome detection approach has been proposed, allowing detection of low counts of exosomes [50-53]. However, label-free detection and quantification of such nanosized objects remains challenging and cumbersome due to the lack of highly sensitive, reproducible, and cost-effective techniques. In particular, the sensitivities of existing optical and plasmonic sensors are not high enough to detect dilute analytes of low molecular weights or low surface coverage of exceedingly small bound molecules [54, 55].

New sensing platforms are needed to address these concerns. To circumvent the limits of plasmonic sensing with traditional materials, research in metamaterials has intensified in the past decade. Metamaterials are a class of engineered materials that do not exist in nature and exhibit exotic and unusual electromagnetic properties that make them attractive for applications in bioengineering and biosensing [56-58]. In particular, metamaterials that show hyperbolic dispersion such as 3D hyperbolic metamaterials (HMMs) and 2D hyperbolic metasurfaces (HMs) have shown extreme sensitivity for low concentrations of smaller bioanalytes [59-62].

HMMs are a class of artificial anisotropic materials, which originates from the concept of optics of crystals. HMM shows hyperbolic dispersion $[63,64]$ because the outof-plane dielectric component $\varepsilon_{z z}=\varepsilon_{\perp}$ has an opposite sign to the in-plane dielectric components $\varepsilon_{x x}=\varepsilon_{y y}=\varepsilon_{\|}$. As is well known, homogenous isotropic materials exhibit elliptical dispersion, with the dispersion relation $k_{x}^{2}+k_{y}^{2}+k_{z}^{2}=\omega^{2} / c^{2}$. However, uniaxial anisotropic materials such as HMMs have a hyperbolic dispersion relation $\left(k_{x}^{2}+k_{y}^{2}\right) / \varepsilon_{z z}+\left(k_{z}^{2}\right) / \varepsilon_{x x}=\omega^{2} / c^{2}$, where the dielectric response is given by the tensor $\bar{\varepsilon}=\left[\varepsilon_{x x}, \varepsilon_{y y}, \varepsilon_{z z}\right]$ [65-67]. This hyperbolic dispersion allows these materials to support waves with infinitely large momentum (bulk plasmon polaritons, BPPs) in the effective medium limit. These waves can propagate inside HMMs, but are evanescent and decay away exponentially in the superstrate [68]. Because the BPP modes are nonradiative with high momentum, they can only be excited using a momentum coupler such as a prism [69] or grating [70], just like in the case of surface plasmon polaritons. It has been shown that the BPP modes of both type I $\left(\varepsilon_{\|}>0, \varepsilon_{\perp}<0\right)$ and type II $\left(\varepsilon_{\|}<0, \varepsilon_{\perp}>0\right)$ HMMs can be excited using either coupling technique and the excited modes showed high quality (Q) factor resonances [70]. Because HMMs support high Q-factor multimode BPP resonances, they can be used to develop ultrasensitive multimode biosensors [71]. In recent times, various HMM-based ultrasensitive biosensors using different interrogation schemes have been proposed for noninvasive liquid biopsies [59-61, 72, 73].

Kabashin et al. first proposed to use type I HMM, such as gold nanorods electrochemically grown into a substrate, in biosensing [59]. This plasmonic nanorod HMM excited a bulk guided mode in the near-infrared (NIR) wavelength range using prism coupling and exhibited a record bulk refractive index (RI) sensitivity of 32,000 $\mathrm{nm}$ per refractive index unit (RIU). They also demonstrated the real-time binding of small biomolecules such as biotin at a concentration as low as $1 \mu \mathrm{M}$. Sreekanth et al. developed a compact multimode biosensor by exciting the BPP modes of an $\mathrm{Au} / \mathrm{Al}_{2} \mathrm{O}_{3} \mathrm{HMM}$ using a grating coupling approach [60]. This miniaturized multimode sensor exhibited extreme bulk RI sensitivity with a record figure of merit (FOM) and detected biotin concentrations as low as $10 \mathrm{pM}$. Since then different hyperbolic dispersion metamaterials have been proposed for sensitivity enhancement purposes [72-82].

One avenue for exploration is using alternative techniques for interrogating the sensor, such as the GoosHanchen shift. This has shown to improve the detection limit for small molecules on a 2D hyperbolic metasurface [61]. Another avenue is the development of HMMs free of noble metals, which would also improve the small molecule detection limit because the performance of plasmonic sensors in the visible frequency range is significantly limited by inherent losses in the metallic components. In this direction, a TiN/ $\mathrm{Sb}_{2} \mathrm{~S}_{3}$ multilayered HMM-based reconfigurable biosensor has been recently proposed [72]. Plasmonic sensors are also hampered by the limitations of 
the biomolecular receptors used to target analytes. The receptors can bind to parasitic molecules that cannot be easily distinguished from the targets, which results in a false positive signal. One way around this issue is the use of multiplex detection, realized by developing HMM-based multimode sensors [60] and reconfigurable sensors [72, 83]. In particular, the sensitivity of these sensors can be tuned from maximum to minimum, so that different molecular weight biomolecules including parasitic molecules can be recognized. However, algorithms to analyze proper logic functions based on this concept must be implemented through deep learning or artificial intelligence [9].

In short, significant progress has been made in realtime label-free biosensing of nanoscale targets - such as small molecules and exosomes - at lower concentrations using HMM-based plasmonic biosensor platforms. These in turn can be used to develop cost-effective, noninvasive liquid biopsies for point-of-care (POC) clinical evaluation, early cancer screening and real-time diagnosis of diseases. This review highlights the recent advances in hyperbolic dispersion metasurfaces-based biosensor technology, and in particular discusses biosensors involving metal/ dielectric multilayered HMMs. This includes biosensors using different coupling mechanisms and interrogation schemes. We also discuss reconfigurable sensors based on tunable HMMs, localized surface plasmon resonance (LSPR) sensors based on type I HMMs, and biosensing applications of multifunctional hyperbolic nanocavities.

\section{Enabling highly tunable engineering of the optical density of states in multifunctional hyperbolic nanocavities for biosensing applications}

HMMs of either type I or type II have been investigated in confined in-plane geometries for a variety of applications. In particular, miniaturized optical cavities made of metaldielectric multilayers have been shown to enable radical increases in the photon density of states. They thus represent an ideal platform to enhance light-matter interactions for applications in optical nanotechnologies [84]. In 2012, Yang et al. reported on nanostructured optical cavities made of indefinite metal-dielectric multilayers that confine the electromagnetic field down to $\lambda / 12$ and with ultrahigh (up to 17.4) optical refractive indices [85] (Figure 1).
Their experiments also revealed that these types of cavities display anomalous scaling laws. For instance, cavities with different sizes can be resonant at the same frequency, and higher-order resonant modes oscillate at lower frequencies. These archetypical structures were also proposed to enhance the quantum yield and spontaneous emission of quantum emitters. In 2014, Guclu et al. proposed a radiative emission enhancement mechanism based on the use of hyperbolic nanostructures [86]. In particular, they showed that hyperbolic metamaterial resonators behave like nanoantennas and, owing to their very large density of states, can lead to a 100 -fold enhancement of the radiative emission of quantum emitters placed in their vicinity. The same year, $\mathrm{Lu}$ et al. demonstrated experimentally enhanced spontaneous emission rates of molecules by using nanopatterned multilayered hyperbolic metamaterials [87] (Figure 2).

They showed that by nanopatterning an HMM of type II made of $\mathrm{Ag}$ and $\mathrm{Si}$ multilayers, the spontaneous emission rate of rhodamine dye molecules is enhanced 76 -fold at tunable frequencies and the emission intensity of the dye increases by a factor 80 compared with the same HMM without nanostructuring. The same group has also

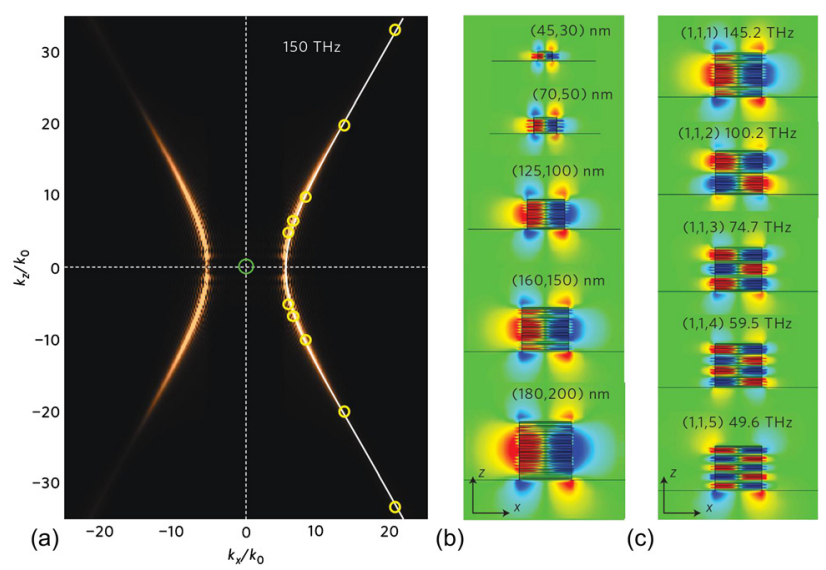

Figure 1: Finite-difference time-domain (FDTD)-calculated isofrequency contour (IFC) of the multilayer metamaterial and mode profiles of indefinite optical cavities. (a) Cross-sectional view of the hyperbolic IFC for $4 \mathrm{~nm}$ silver and $6 \mathrm{~nm}$ germanium multilayer metamaterial at $150 \mathrm{THz}$ (bronze curve), which matches the effective medium calculation (white line). The yellow circles represent the resonating wave vectors of the cavity modes shown in $b$, and the green circle represents the light cone of air. (b) FDTD-calculated electric field $\left(E_{z}\right)$ distributions of the $(1,1,1)$ mode for cavities made of $4 \mathrm{~nm}$ silver and $6 \mathrm{~nm}$ germanium multilayer metamaterial with different size (width, height) combinations but at the same resonant frequency of $150 \mathrm{THz}$. (c) FDTD-calculated $\left(E_{z}\right)$ distributions of the first five cavity modes along the $z$-direction for the $(160,150) \mathrm{nm}$ cavity. Reproduced with permission [85]. Copyright 2012, Nature Publication Group. 

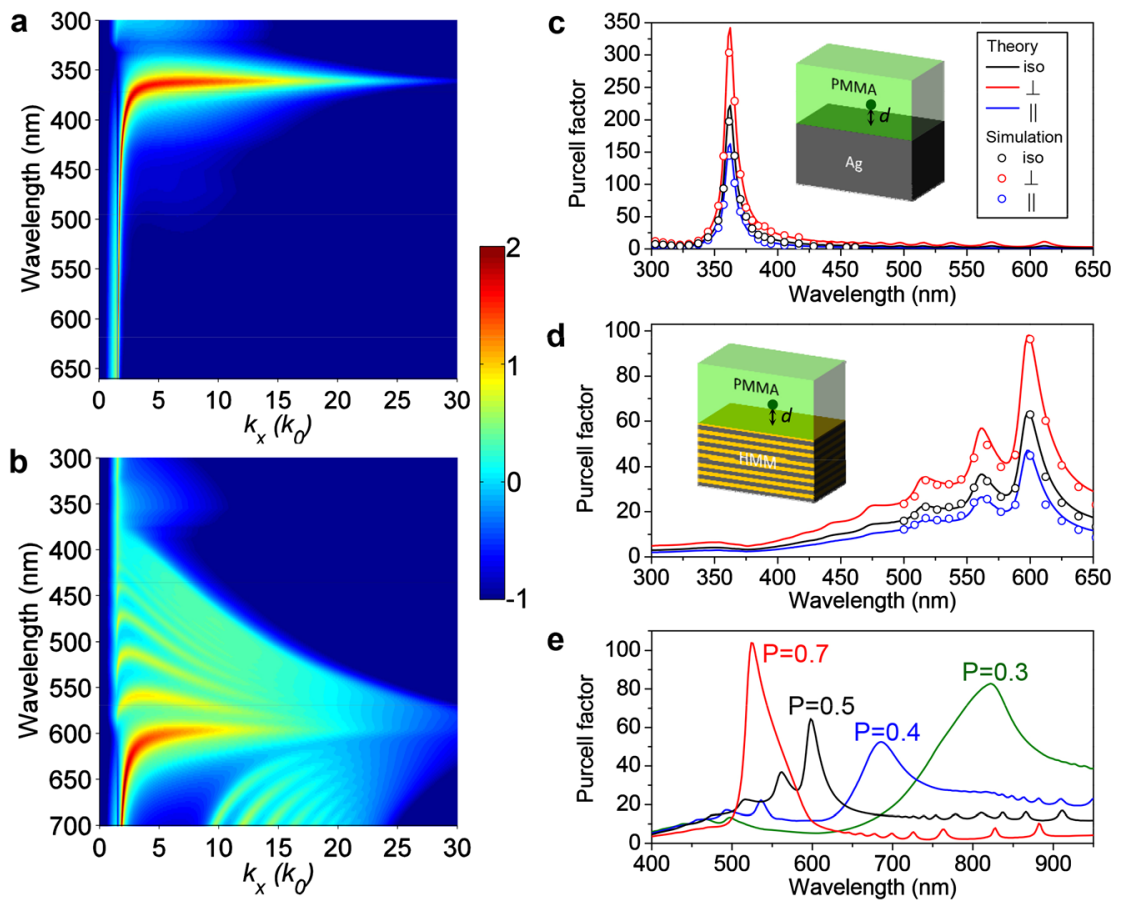

Figure 2: Comparison of Purcell factors for Ag-Si multilayer hyperbolic metamaterials (HMMs) and a pure Ag single layer. a, b, Normalized dissipated power spectra (intensity on a logarithmic scale) for a dipole perpendicular to and at a distance of $d=10 \mathrm{~nm}$ above a uniform Ag single layer (a) and a Ag-Si multilayer HMM (b), each with the same total thickness of $305 \mathrm{~nm}$. The multilayer has 15 pairs of Ag and Si layers (each layer thickness is $10 \mathrm{~nm}$ ). The color scales indicate normalized dissipated power. (c, d) Purcell factor for a dipole located $d=10 \mathrm{~nm}$ above the uniform Ag single layer (c) and the Ag-Si multilayer HMM (d), as depicted in the insets. The Purcell factor for isotropic dipoles (iso, black lines) is averaged from that of the dipoles perpendicular ( $\perp$, red lines) and parallel (\|, blue lines) to the surface. Corresponding threedimensional full-wave simulations (open circles) agree with theoretical calculations. (e) Tunable Purcell enhancement across the visible spectra for isotropic dipoles located $d=10 \mathrm{~nm}$ above the uniform Ag-Si HMMs by adjusting the volumetric filling ratio of the metal, P. Reproduced with permission [87]. Copyright 2014, Nature Publication Group.

reported a 160-fold enhancement of the spontaneous carrier recombination rates in InGaN/GaN quantum wells thanks to the excitation of hyperbolic modes supported by stacking Ag-Si multilayers, which enable a high tunability in the plasmonic density of states for enhancing light emission at various wavelengths [88]. This approach led to the realization of ultrafast and bright quantum well-based LEDs with a 3-dB modulation bandwidth beyond $100 \mathrm{GHz}$. More recently, Indukuri et al. proposed nanostructured hyperbolic antennas with an engineered optical density of states and a very large quality-factor/modal volume ratio. Their architecture can enable applications in cavity quantum electrodynamics, nonlinear optics, and biosensors [89]. From a more fundamental point of view, research have been recently focused on layered metal-dielectric HMMs, which support a wide landscape of surface plasmon polaritons and Bloch-like gap-plasmon polaritons with high modal confinement. Light can excite only a subset of these modes, and typically within a limited energy/momentum range if compared with the large set of high- $k$ modes supported by hyperbolic dispersion media, and coupling with gratings
[90-92] or local excitation [93] is necessary. Isoniemi et al. recently used electron energy loss spectroscopy (EELS) to achieve a nm-scale local excitation and mapping of the spatial field distribution of bright and dark modes in multilayered type II HMM nanostructures, such as HMM pillars and HMM slot cavities [94] (Figure 3). In particular, as recently demonstrated by Maccaferri et al., HMM nanoantennas can enable a full control of absorption and scattering channels [95].

By varying the geometry of the nanoantennas, the ratio of scattering and absorption and their relative enhancement/quenching can be tuned over a broad spectral range from visible to NIR. Notably, both radiative and nonradiative modes supported by this type of architecture can be excited directly with far-field radiation, even when the radiative channels are almost totally suppressed. Similarly, Song and Zhou showed that multiresonant composite nanoantennas made of vertically stacked building blocks of metal-insulator loop nanoantennas can support multiple nanolocalized modes at different resonant wavelengths, and are thus suitable for multiband operations, 


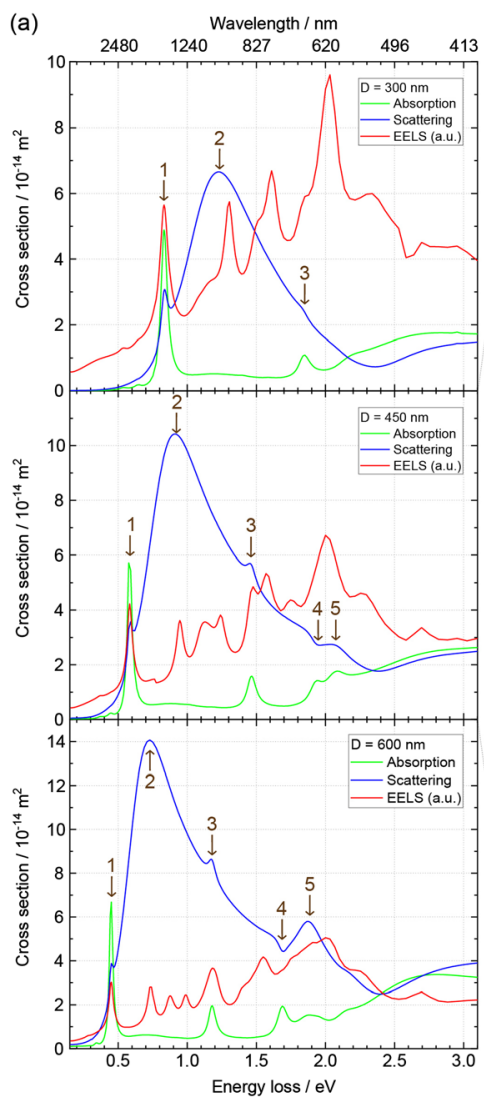

(b)
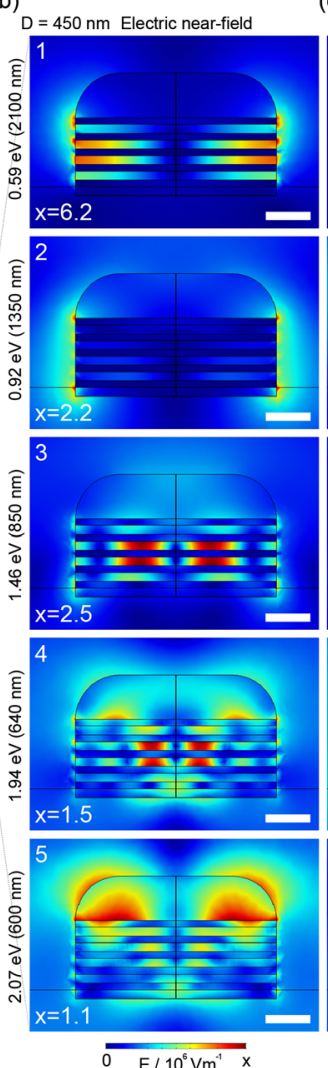

(c)

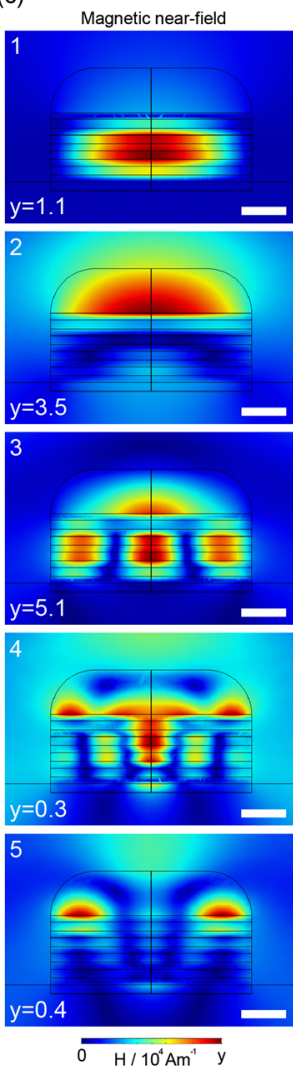

Figure 3: (a) Simulated optical absorption and scattering spectra of hyperbolic metamaterial (HMM) pillars with three different diameters compared with Electron Energy Loss Spectroscopy (EELS) simulations using a vertical beam. Absorption and scattering cross sections are comparable between each other and between pillar sizes, whereas the EEL spectra are comparable only between pillar sizes, using arbitrary units. (b) Electric and (c) magnetic near-field intensities in the $450 \mathrm{~nm}$ pillar using plane wave excitation with marked energies. The plane wave propagates from the top, along the axis of symmetry of the pillar. The corresponding resonances are marked with numbered arrows in a, and resonances with similar field profiles for other pillar diameters are marked with the same number. The maximum value of the color scale is marked at lower right in each plot. Scale bars $=100 \mathrm{~nm}$. Reproduced with permission [94]. Copyright 2020, Wiley-VCH. such as multiphoton processes, broadband solar energy conversion, and wavelength multiplexing [96] (Figure 4).

Furthermore, these hyperbolic nanoantennas have proven to possess both angular and polarizationindependent structural integrity, unlocking promising applications as solvable nanostructures. In this framework, Wang et al. experimentally synthetized and measured the optical properties of hyperbolic nanoparticles made with either Au or dielectric nanoobjects coated with alternating $\mathrm{SiO}_{2}$ and $\mathrm{Au}$ multishells [97]. These soluble nanoparticles

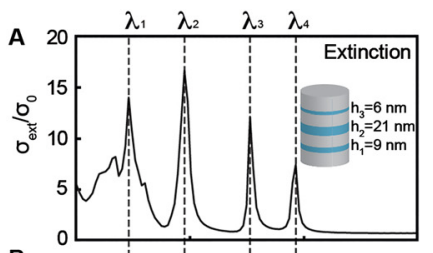

B

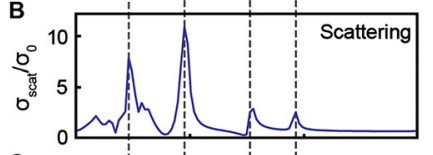

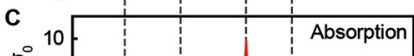

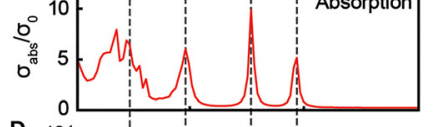

D

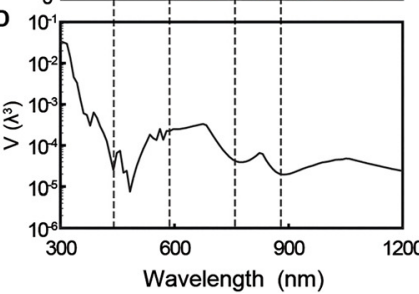

E $\quad \lambda_{1}=439 \mathrm{~nm}$
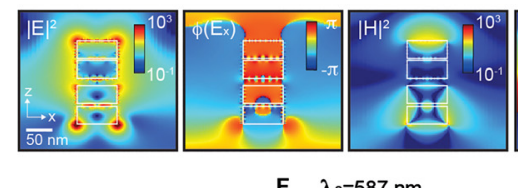

F $\quad \lambda_{2}=587 \mathrm{~nm}$
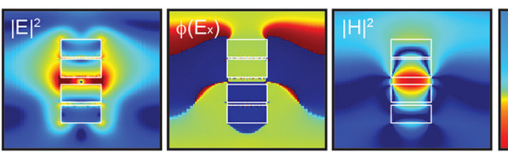

G $\lambda_{3}=762 \mathrm{~nm}$
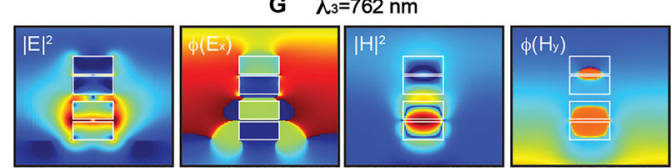

H $\lambda_{4}=884 \mathrm{~nm}$
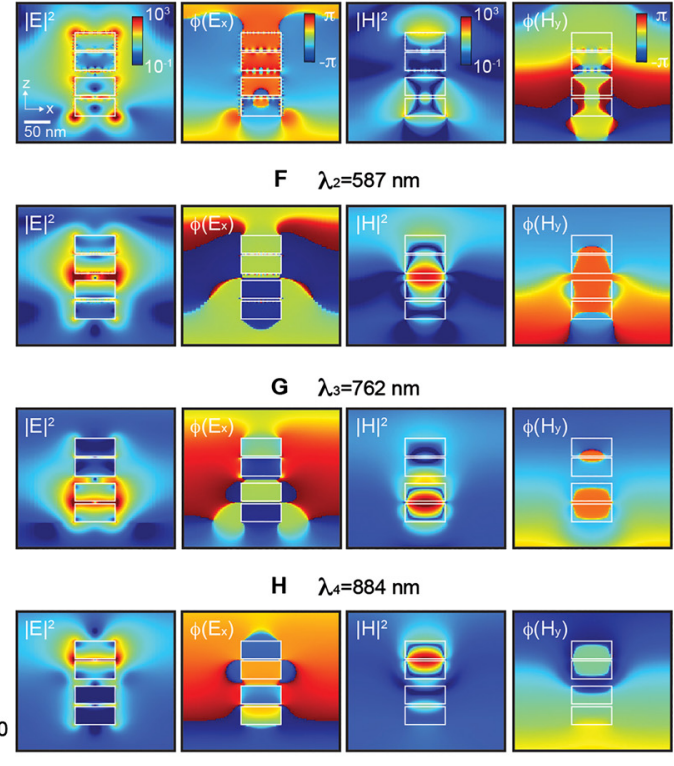

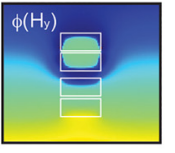

Figure 4: Far-field and near-field optical properties of multiresonant composite nanoantennas. FDTD-calculated spectra of (a) normalized extinction cross-section $\sigma_{\text {ext }} / \sigma_{0}$, (b) normalized scattering crosssection $\sigma_{\text {sca }} / \sigma_{0}$, (c) normalized absorption cross-section $\sigma_{\mathrm{abs}} / \sigma_{0}$, and (d) normalized mode volume $V_{m} / \lambda^{3}$ for a meta-

l-insulator-metal-insulator-metal-insulator-metal (MIMIMIM) composite nanoantenna with three dielectric layers under linear polarized plane wave excitation at normal incidence angle. (e-h) FDTD-calculated distribution maps of electric field intensity $|E|^{2}$, phase of in-plane electric field $\Phi\left(E_{\chi}\right)$, magnetic field intensity | $\left.H\right|^{2}$, and phase of magnetic field $\Phi\left(H_{y}\right)$ for MIMIMIM composite nanoantenna at resonant wavelengths of (e) $439 \mathrm{~nm}$, (f) $587 \mathrm{~nm}$, (g) $762 \mathrm{~nm}$, and (h) $884 \mathrm{~nm}$. Reproduced with permission [96]. Copyright 2018, American Chemical Society. 
enable highly tunable optical modes covering a broad wavelength range. Moreover, they exhibit high local field intensity enhancement, thus opening excellent opportunities in plasmon-enhanced spectroscopy, nanolasers, design of nonlinear phenomena, photothermal conversions, and hot-electron generation. It is worth mentioning here that nanostructured HMMs have also been proposed to achieve a near-perfect absorption of light. Zhou et al. have proposed a broadband absorber based on a tapered a hyperbolic metamaterial, made of alternating metal-dielectric

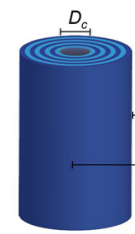

(a)

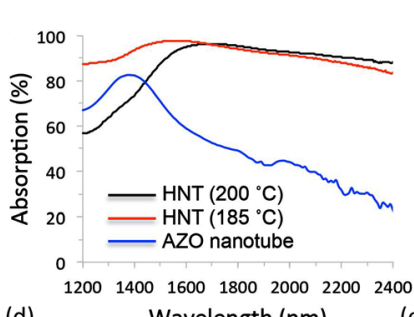

(d)

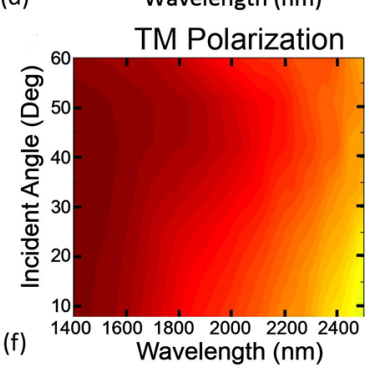

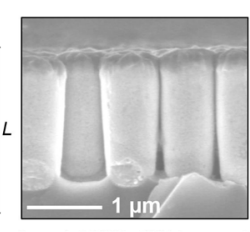

(b)

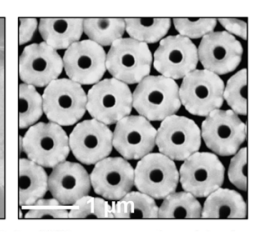

(c) (e)
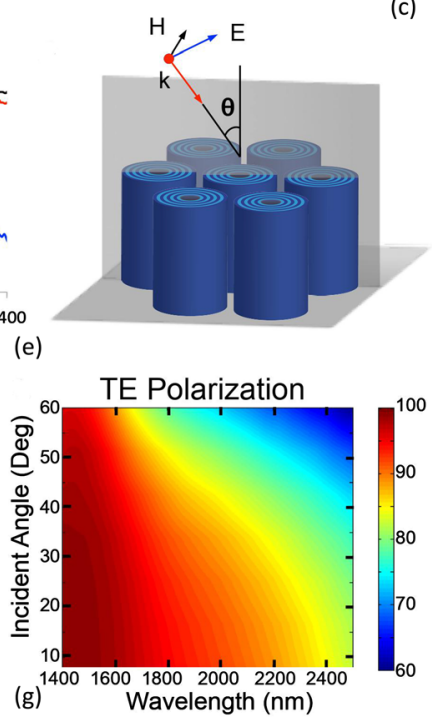

Figure 5: Experimental realization of the hyperbolic metamaterial (HMM) broadband absorber targeting the visible and IR range. (a) Sketch of an array of metal-dielectric multilayered tapers under a transverse magnetic (TM) wave incidence from the top. (b-d) Measured (black) and simulated (red) absorption curves for broadband absorbers with SEM images (inset): (b) $700 \mathrm{~nm}$ array period, 9-stack structure $\left(\mathrm{Au}-\mathrm{Al}_{2} \mathrm{O}_{3}\right)$ absorbing from 1.5 to $3 \mu \mathrm{m}$; (c) $700 \mathrm{~nm}$ array period, 11-stack structure $(\mathrm{Au}-\mathrm{Ge})$ absorbing from 2.5 to $6 \mu \mathrm{m}$; (d) $220 \mathrm{~nm}$ period, three-stack structure $\left(\mathrm{Al}-\mathrm{SiO}_{2}\right)$ absorbing from $\approx 0.4$ to $1.2 \mu \mathrm{m}$, including angled data at $45^{\circ}$ (dashed blue curve). Scale bars represent 500, 500, and $200 \mathrm{~nm}$ from b to d. (e) Simulated absorption spectra of a multilayered structure and its effective medium counterpart under a TM wave incidence. The multilayered structure is an array of $\mathrm{Ag}(20 \mathrm{~nm}) / \mathrm{Ge}(30 \mathrm{~nm})$ multilayered, tapered HMM waveguides with a height of $900 \mathrm{~nm}$, a tapering width from $500 \mathrm{~nm}$ at the bottom to $182 \mathrm{~nm}$ at the top, and a period of $700 \mathrm{~nm}$. (f) Sketch of the isofrequency contour (IFC) of the multilayered structure (blue) compared with that of the effective medium (red). The dashed blue curve represents the IFC of the multilayered structure at a smaller wavelength. Reproduced with permission [101]. Copyright 2017, National Academy of Sciences. multilayers and working in the visible and NIR ranges [98] (Figure 5).

They demonstrated that light couples into the tapered structure most strongly due to the hyperbolic dispersion phase-matching conditions. Moreover, they obtain a broad absorption band thanks to the broadening of the resonances from an array of coupled HMM tapered structures. Similarly, Sakhdari et al. theoretically propose a vertically integrated hot-electron device based on a nanostructured HMMs that can efficiently couple plasmonic excitations into electron flows, with an external quantum efficiency approaching the physical limit [99]. Their metamaterialbased architecture displays a broadband and omnidirectional response at infrared and visible wavelengths, thus representing a promising platform for energy-efficient photodetection and energy harvesting beyond the bandgap spectral limit. More recently, Abdelatif et al. proposed a funnel-shaped anisotropic metamaterial absorber made of periodic array of nickel-germanium (Ni/ $\mathrm{Ge}$ ) enabling enhanced broadband absorption (up to 96\%) due to the excitation of multiple orders of slow-light modes over a wavelength range from the ultraviolet to NIR range [100]. In 2017, Riley et al. have synthetized transferrable hyperbolic metamaterial particles showing broadband, selective, omnidirectional, perfect absorption. Their system is made of hyperbolic nanotubes (HNTs) on a silicon substrate that exhibit near-perfect absorption at telecommunication wavelengths even after being transferred to a mechanically flexible, visibly transparent polymer, which is a more desirable substrate in view of mechanically flexible and low-cost applications [101] (Figure 6).

Finally, Caligiuri et al. presented an approach to achieve super-absorption capabilities based on resonant gain singularities. The proposed mechanism enables a huge amplification of the emitted photons resonantly interacting with a multilayered hyperbolic system. In particular, they theoretically demonstrated that metal/doped-dielectric multishell nanostructures can be used as self-enhanced loss compensated devices, being a favorable scenario for low-threshold SPASER action [102].

\section{Extreme sensitivity biosensing platform based on HMMs}

Hyperbolic metamaterials support both radiative and nonradiative modes. The radiative modes such as FerrellBerreman $[103,104]$ and Brewster modes $[75,105]$ can be excited from free space. However, a momentum-matching condition must be satisfied to excite HMM nonradiative 
(a)

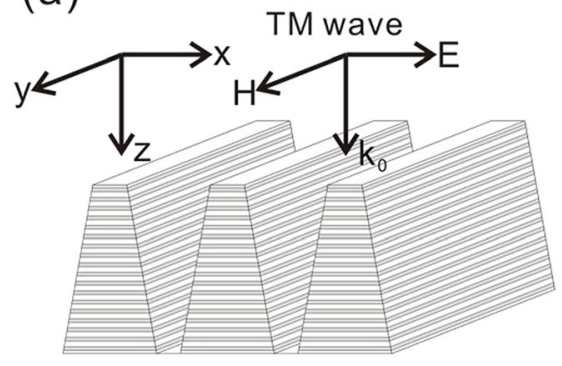

(c)

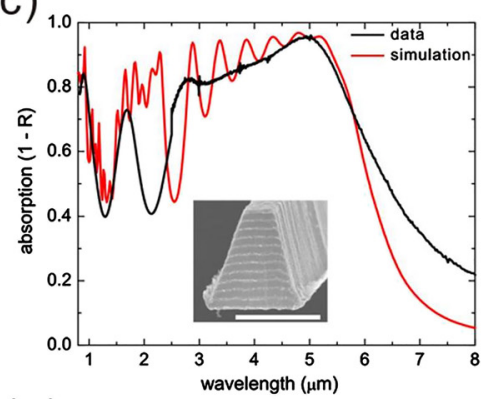

(e)

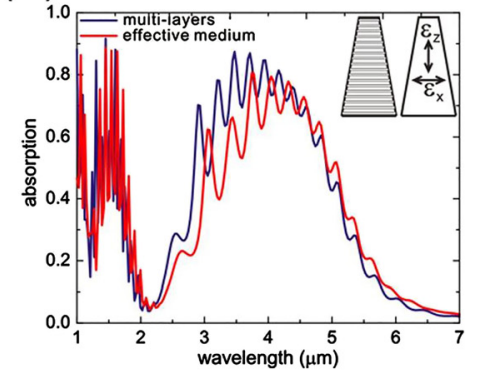

(b)

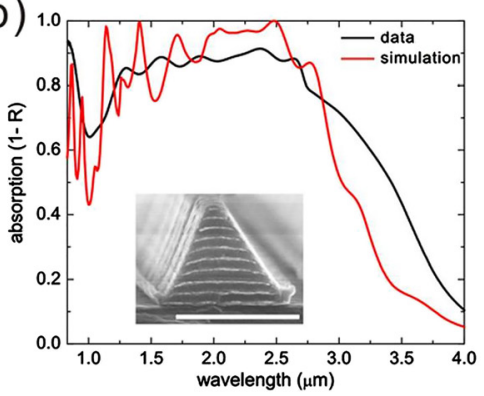

(d)

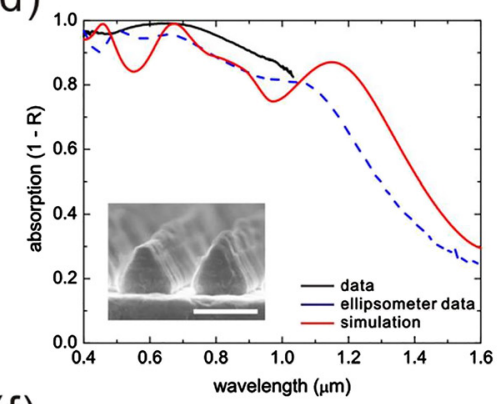

(f)

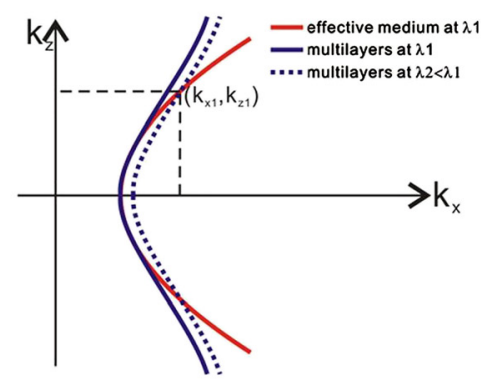

Figure 6: (a) Scanning electron microscopy (SEM) of coupled hyperbolic nanotubes (HNTs). SEM images of the HNT arrays as viewed in the plane (b) parallel and (c) perpendicular to the nanotube axis. (d) Absorption spectra of HNT arrays deposited at a temperature of 185 and $200{ }^{\circ} \mathrm{C}$ along with a spectrum of a pure aluminum-doped zinc oxide (AZO) nanotube array deposited at $200^{\circ} \mathrm{C}$. (e) Schematic of the incident radiation at angle $\theta$ showing TM polarization. Wide-angle absorption spectra for (f) TM and (g) TE polarizations of an HNT array deposited at $200^{\circ} \mathrm{C}$. The color corresponds to the percentage absorption. Reproduced with permission [98]. Copyright 2014, American Chemical Society. modes, such as SPP and BPP modes. A phase-sensitive HMM-based biosensor can be developed by exciting the Brewster mode of the HMM because a sudden phase jump occurs at the Brewster angle. In this scheme, the phase shift at the Brewster angle due to the change in RI arising from the presence of the analyte can be recorded as the sensing parameter [75]. However, extreme sensitivity biosensing is only possible by exciting the BPP modes of the HMM. A type II HMM supports only high- $k$ BPP modes, whereas a type I HMM supports both high- $k$ and low- $k$ BPP modes. In the following sections, we discuss the development of multilayered HMM-based biosensors based on metasurface grating and prism coupling excitation schemes.

\subsection{Grating-coupled HMM-based multimode biosensor}

In this section, the excitation of BPP modes of type II HMMs using grating coupling and its application for the development of biosensors with differential sensitivity is reported. Wavelength and angular interrogation schemes to demonstrate the extreme sensitivity of the biosensor for small molecule detection at low analyte concentrations have been investigated [73, 106]. The HMM-based plasmonic biosensor platform, with integrated microfluidics, is illustrated in Figure 7a and b. As can be seen, it is a combination of a plasmonic metasurface and an HMM. The HMM consists of 16 alternating thin layers of gold and aluminum dioxide $\left(\mathrm{Al}_{2} \mathrm{O}_{3}\right)$ with thickness 16 and $30 \mathrm{~nm}$, respectively. The fabricated multilayer is a type II HMM, which shows hyperbolic dispersion at $\lambda \geq 520 \mathrm{~nm}$, where the real parts of parallel and perpendicular permittivity components are negative and positive, respectively (Figure 7c). To excite the BPP modes of $\mathrm{Au}-\mathrm{Al}_{2} \mathrm{O}_{3} \mathrm{HMM}$, the grating coupling technique has been used [70, 107, 108]. For this purpose, a 2D subwavelength $\mathrm{Au}$ diffraction grating with period $500 \mathrm{~nm}$ and hole dimeter $160 \mathrm{~nm}$ was integrated with the HMM (inset of Figure 7a). Au has been considered because it is the most popular plasmonic 
(a)
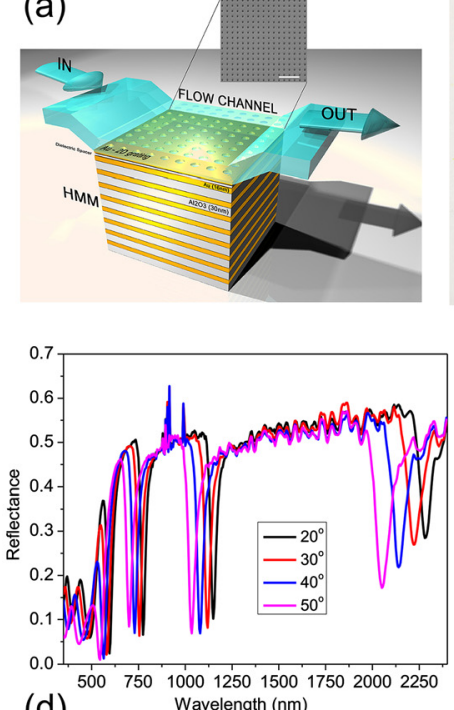

(d)
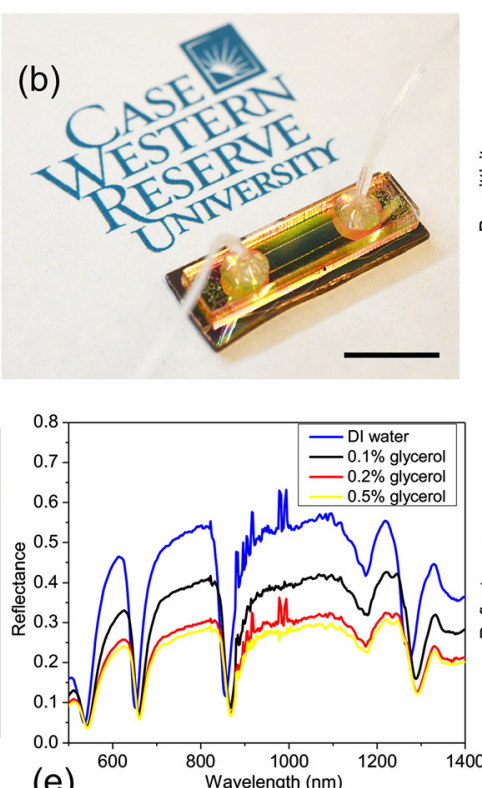

(e)
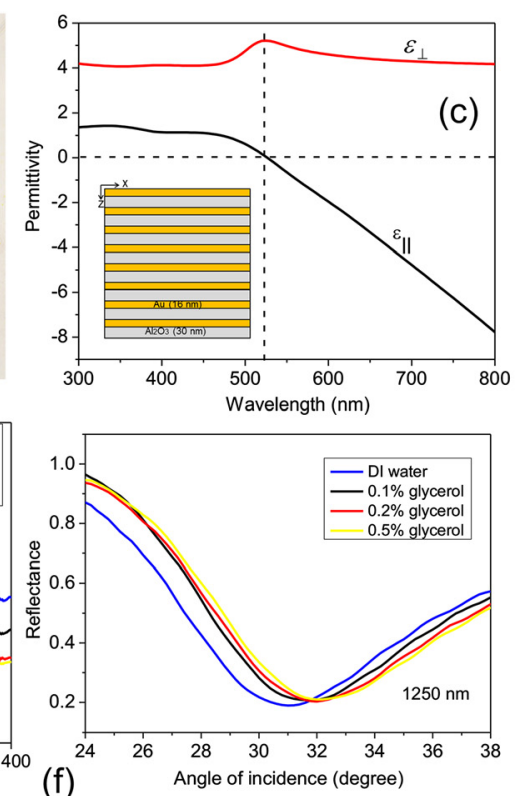

Figure 7: (a) A schematic diagram of the fabricated grating-coupled HMM (GC-HMM)-based sensor device and an SEM image of the 2D subwavelength Au diffraction grating on top the HMM. (b) A photograph of the sensor device. Scale bar $=10 \mathrm{~mm}$. (c) Calculated real parts of effective permittivity for an $\mathrm{HMM}$ consisting of eight pairs of gold $/ \mathrm{Al}_{2} \mathrm{O}_{3}$ layers determined using effective media theory. (d) Excited BPP modes of the GC-HMM at different angles of incidence. Reproduced with permission from Sreekanth et al. [60], Copyright 2016, Springer Nature Publication Group. (e, f) Standard sensor calibration tests, injecting different weight percentages of glycerol $(0.1-0.5 \% \mathrm{w} / \mathrm{v})$, using wavelength interrogation (e) and angular interrogation (f). Reproduced with permission from Sreekanth et al. [73], Copyright 2017, EDP Sciences.

material for biosensing applications because of its low oxidation rate and high biocompatibility.

The principle behind grating coupling is that the surface plasmons supported by a metasurface grating can be excited when the parallel wavevectors of the surface plasmons are comparable with the wavevector of the light. Grating diffraction orders are no longer propagating waves when this condition is satisfied, but they are evanescent fields. The enhanced wavevector of the evanescent field is responsible for the coupling of incident light to the surface plasmon modes $[109,110]$. The proposed metasurface grating-coupled HMM (GC-HMM)-based sensor works based on the coupling condition between the metasurface grating modes and BPP modes. The coupling condition alters when the RI of the surrounding medium changes, which allows measuring a change in resonance wavelength and resonance angle. Explicitly, the grating coupling condition is given by $k_{\mathrm{SPP}}=n_{0} k_{0} \sin \theta \pm m k_{g}$, where $\theta$ is the incident grazing angle, $n_{0}$ is the RI of surrounding medium, $k_{0}=2 \pi / \lambda$ is the vacuum wavevector, $m$ is the grating diffraction order, and $k_{g}=2 \pi / \Lambda$ is the grating wavevector with $\Lambda$ being the grating period.

To show the BPP modes of the $\mathrm{Au}-\mathrm{Al}_{2} \mathrm{O}_{3} \mathrm{HMM}$, the reflectance spectra of the GC-HMM with varying incidence angle, have been acquired using a variable angle highresolution spectroscopic ellipsometer. The $\mathrm{Q}$ factors of these modes are remarkably high because of strong mode confinement and large modal indices. In Figure $7 \mathrm{~d}$, the spectral response of the fabricated sensor at different angles of incidence has been shown. The narrow modes recorded at wavelengths above $500 \mathrm{~nm}$ represent highly confined fundamental and higher-order BPP modes of the HMM. The Q-factors of the modes measured at resonance wavelengths 1120,755 , and $580 \mathrm{~nm}$ are $29.5,26$, and 23 , respectively. The sensor supports many modes with different Q-factors, which increases with increasing mode resonance wavelength.

The performance of the HMM-based sensor using spectral and angular interrogation schemes has been analyzed. The detection limit of the sensor has been first determined by injecting different weight ratios of aqueous solutions of glycerol into the sensor microchannel (sample volume $=1.4 \mu \mathrm{l}$ ). In Figure 7e and f, the reflectance spectra of the sensor with varying concentrations of glycerol in distilled water $(0.1-0.5 \% \mathrm{w} / \mathrm{v})$ have been shown, using spectral and angular scans, respectively. It is evident from Figure $7 \mathrm{e}$ that the resonance wavelength corresponding to each BPP mode red shifts and the quality factor of each mode declines with increasing glycerol concentration. In the angular scan, a positive angular shift is obtained when the glycerol weight ratio is increased (Figure $7 f$ ). The sensor can record extremely small RI changes of glycerol concentrations in both scans. For example, a significant shift 
of $12 \mathrm{~nm}$ is obtained at $1300 \mathrm{~nm}$ even with $0.1 \% \mathrm{w} / \mathrm{v}$ glycerol concentration. It should be noted that the shift increases when the spectral position of the BPP mode varies from visible to NIR wavelengths because the transverse decay of the field in the superstrate strongly varies from one mode to another. Thus the sensor provides different sensitivities as the resonance wavelength increases from visible to NIR wavelengths due to the differential response of the BPP modes. The measured maximum bulk RI sensitivity for the longest wavelength BPP mode in spectral and angular scans is around 30,000 nm/RIU and $2500^{\circ} \%$ RIU, respectively. The minimum sensitivity is recorded at the shortest wavelength BPP mode, which is $13,333 \mathrm{~nm} / \mathrm{RIU}$ and $2333^{\circ}$ /RIU for spectral and angular scans, respectively. In addition, the sensor exhibits different FOM for each BPP mode, which are 206, 357, 535 and 590 at 550, 660, 880, and $1300 \mathrm{~nm}$, respectively. It is worth noting that the recorded FOMs of the proposed multilayered HMM-based sensor are much higher than existing plasmonic biosensors. An interesting characteristic of the proposed sensor is the flexibility in the selection of a mode for the identification of specific biomolecules since it provides differential sensitivities.

To demonstrate the capabilities of the proposed sensor device for the detection of smaller biomolecules, biotin (molecular weight, $244 \mathrm{Da}$ ) has been selected as the analyte because it is a model system for small molecule compounds such as vitamins, cancer-specific proteins, hormones, therapeutics, or contaminants such as pesticides or toxins. To selectively detect biotin, the sensor surface has been immobilized with streptavidin biomolecules and the sensor monitors the wavelength or angular shifts due to the RI change caused by the capture of biotin at the streptavidin sites. The sensor performance is monitored by injecting different concentrations $(10 \mathrm{pM}-10 \mu \mathrm{M})$ of biotin prepared in PBS into the sensor microchannel. The reflectance spectra of the sensor have been recorded after a reaction time of $40 \mathrm{~min}$ for each concentration of biotin. PBS was introduced into the microchannel to remove the unbound and weakly attached biotin molecules before each injection of a new concentration of biotin. In Figure 8a and $\mathrm{b}$, the responses of the device during the detection of different concentrations of biotin in spectral and angular scans have been plotted respectively. One can see that an increase in spectral and angular shift is obtained with increasing biotin concentrations. It reflects the increase in RI change due to the capture of biotin molecules on the streptavidin sites. Moreover, a nonlinear variation in shift with increase in biotin concentration is observed in both scans. In Figure 8c, the real-time binding kinetics by
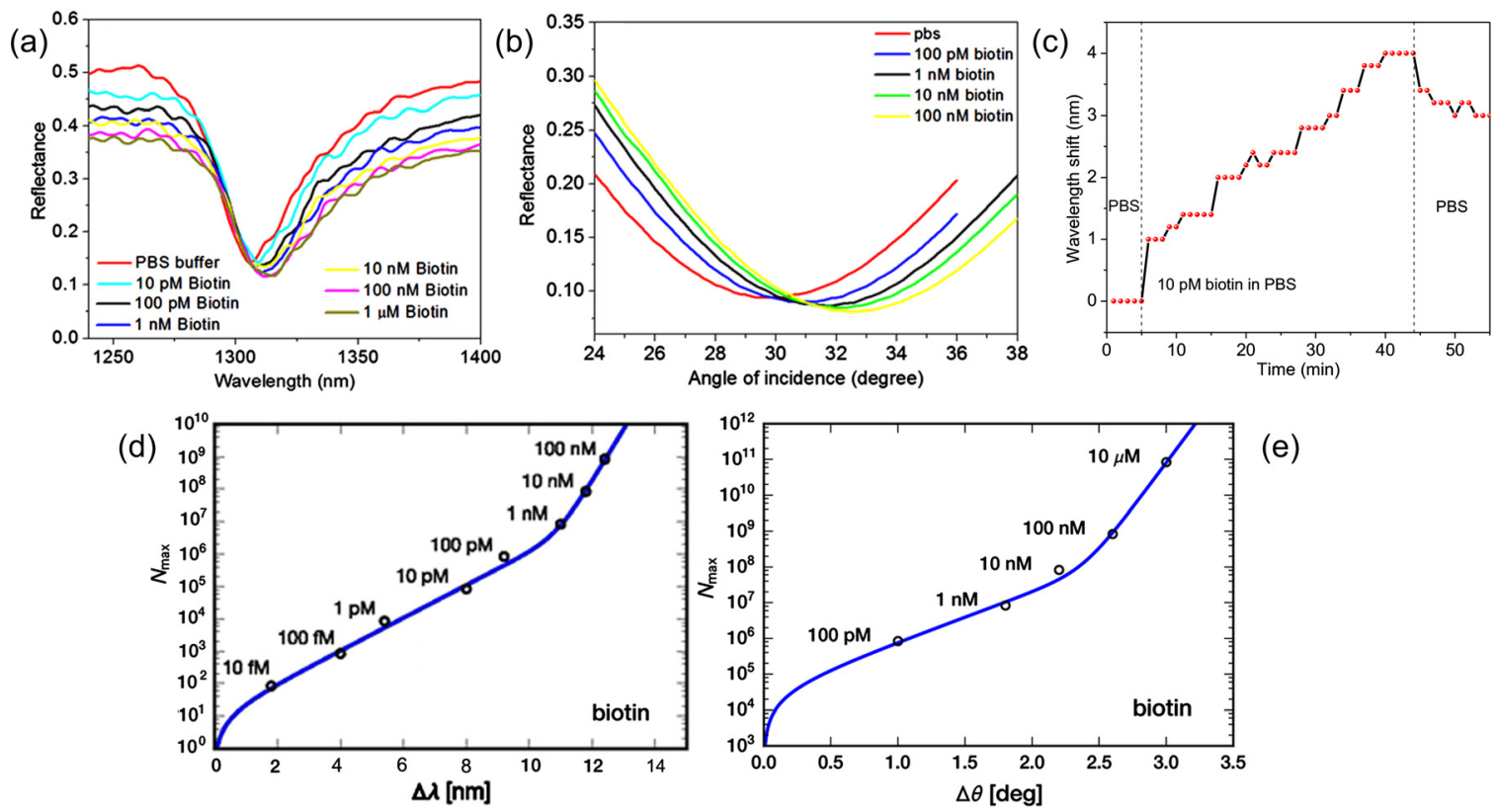

(e)

Figure 8: Detection of biotin using the grating-coupled HMM (GC-HMM)-based sensor. (a) Sensor reflectance spectra as a function of wavelength with different concentrations of biotin in PBS at $30^{\circ}$ angle of incidence. (b) Sensor reflectance spectra as a function of incident angle with different concentrations of biotin in PBS at wavelength $1250 \mathrm{~nm}$. (c) Demonstration of real-time binding of biotin by injecting $10 \mathrm{pM}$ biotin in PBS (in the time interval between the two dotted lines). (d, e) For the mode located at $1280 \mathrm{~nm}$, the maximum number of biotin molecules adsorbed in the illuminated sensor area versus the corresponding wavelength shift $\Delta \lambda$ (d) and versus the corresponding angular shift $\Delta \theta$ (e) at different biotin concentrations. Reproduced with permission from Sreekanth et al. [73], Copyright 2017, EDP Sciences. 
injecting $10 \mathrm{pM}$ biotin has been shown, where a red shift has been observed and discrete steps in resonance wavelength over time, which is due to the $0.2 \mathrm{~nm}$ discreteness in the wavelength sensitivity. Variability in the step size was observed, which is due to statistical fluctuations in which larger or smaller numbers of binding events can occur. Finally, the sensitivity of the wavelength and angular shift to the number of adsorbed biotin molecules on the sensor surface have been investigated. The saturation values of the wavelength $(\Delta \lambda)$ and angular $(\Delta \theta)$ shift for each concentration $c$ of biotin in PBS were considered. Because the shift of the resonance wavelength and angle depends on the number of bound molecules, it is possible to reliably estimate an upper bound $N_{\max }(c)$ based on the sensor parameters. In Figure $8 \mathrm{~d}$ and e, the number of adsorbed biotin molecules with corresponding shifts in wavelength and angle has been plotted, respectively. This behavior is accurately reproduced using a phenomenological doubleexponential fitting function [60]. In contrast to type I HMM-based sensor [59], the proposed multilayered type II HMM-based sensor demonstrates the detection of $10 \mathrm{pM}$ biotin in PBS, which shows that sensitivity is increased by six orders of magnitude.

\subsection{Biomolecular sensing at the interface between chiral metasurfaces and HMMs}

The interface between a chiral metasurface and hyperbolic metamaterials can enable both high sensitivity and specificity for low-molecular-weight nucleic acids and proteins [111-115]. Interestingly, an adapted out-of-plane chiral metasurface enables three key functionalities of the HMM sensor: (i) an efficient diffractive element to excite surface and bulk plasmon polaritons [76, 109]; (ii) an increase in the total sensing surface enabled via out-of-plane binding, improving diffusion-limited detection of small analyte concentrations; and (iii) additional biorecognition assays via circular dichroism (CD) and chiral selectivity that can be optimally tailored to amplify the chiral-chiral interactions between the metamaterial inclusions and the molecules, enabling high-sensitivity handedness detection of enantiomers [116-120].

A sketch of a chiral metasurface hypergrating $(\mathrm{CMH})$, consisting of a periodic array of right-handed Au helices on a type II HMM composed by indium tin oxide (ITO - $20 \mathrm{~nm}$ ) and silver $(\mathrm{Ag}-20 \mathrm{~nm})$ is shown in Figure 9a. From top to bottom, the geometry consists of a superstrate containing the Au helices, the multilayer system (gray stack of ITO/ $\mathrm{Ag}$ ), and the glass substrate. The reflectance and transmittance curves are calculated for a transverse magnetic
(TM) wave and an incident angle $\theta_{i}=50^{\circ}$ with respect to the helix axes. This is done by solving the frequency-domain partial differential equation that governs the $\mathbf{E}$ and $\mathbf{H}$ fields associated with the electromagnetic wave propagating through the structure [121]. As shown in Figure 9b, for the calculated reflectance for the CMH-HMM, it is possible to distinguish three minima at 635 (mode A), 710 (mode B), and $890 \mathrm{~nm}$ (mode C), corresponding to different BPP modes of the underlying HMM, whereas the transmittance remains zero in the entire spectral region.

The sensitivity of the sensing platform can be evaluated as a function of the analyte concentration binding at the chiral metasurface. To this end, different molar fractions of an aqueous solution of 1,2,3-propantriol, characterized by an ultralow molecular weight $\left(\mathrm{C}_{3} \mathrm{H}_{8} \mathrm{O}_{3} \approx 60 \mathrm{Da}\right)$ [122], have been considered. Figure 9b shows the calculated reflection spectra of the CMH-HMM sensor in measuring 1,2,3-propantriol solutions with different concentrations. As expected, the calculated reflectance minima (mode dips) of the coupled system linearly shift toward longer wavelengths with the increase of $\mathrm{RI}$ - from 1.333 (water) to 1.401 (molar fraction of $\mathrm{C}_{3} \mathrm{H}_{8} \mathrm{O}_{3}$ of about $17 \%)$. The corresponding limit of detection (LOD) is equal to $1.5 \times 10^{-4}$ RIU.

An important aspect of the 3D chiral metasurface is the significant increase of the out-of-plane sensing surface because the specific binding of the analytes can occur on the entire helical surface. At the same time, a chiral structure can modify the fluid dynamics around it, inducing an increase of the probability of specific binding.

Clearly, the wavelength shift of BPP modes is strongly related to the quantity of molecules that bind selectively on the surface of the helices. It is possible to quantify this effect by considering different surface coverage of the helix, by considering the maximum RI change equals to 0.068 . Coverage was varied from 0 to $100 \%$, where in the latter case the whole surface of the helix is totally covered by molecules; here a maximum spectral shift of $15 \mathrm{~nm}$ for mode $\mathrm{A}, 21 \mathrm{~nm}$ for mode $\mathrm{B}$, and $31 \mathrm{~nm}$ for mode $\mathrm{C}$ have been obtained, as seen in Figure 9c. The minimum detectable surface coverage, necessary to have an appreciable shift of all three modes, is about $16 \%$, whereas for the most sensitive mode (C) alone, it is approximately $12 \%$.

The sensitivity of the CMH-HMM sensor is also strongly affected by the distance of the bond analyte from the HMM surface. In particular, the local change of RI in a small disk surrounding the helix produces appreciable shifts even when the binding is confined exclusively to the upper region of the helix, which represents the maximum distance from the HMM. In Figure 9d a small disk, with $n=1.401$ and corresponding to an adsorbed surface of $20 \%$, 


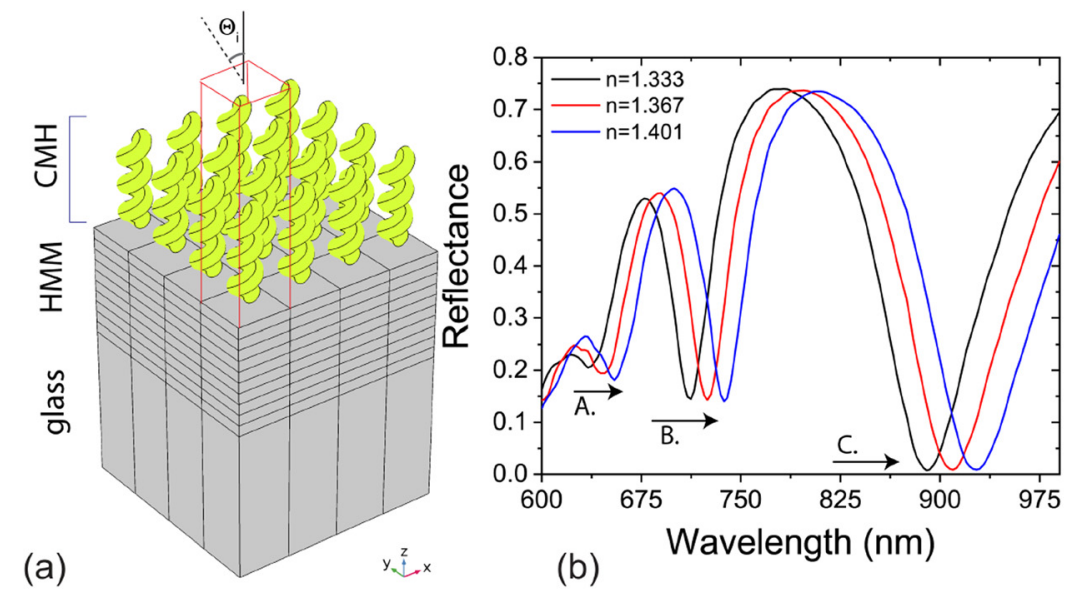

(a)
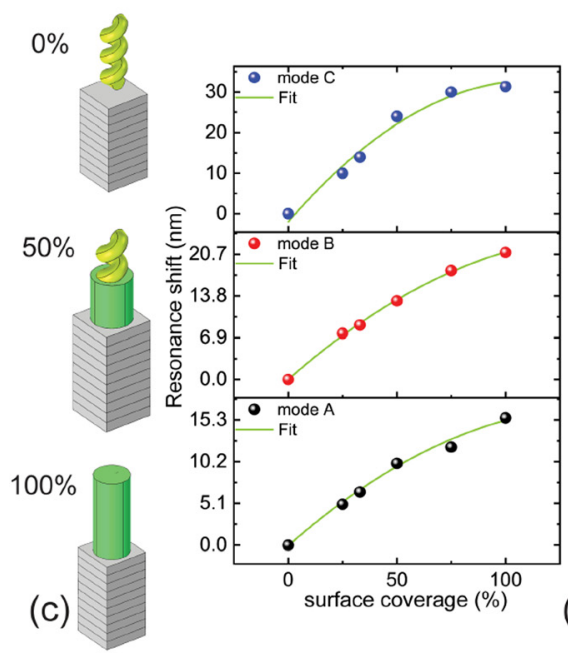

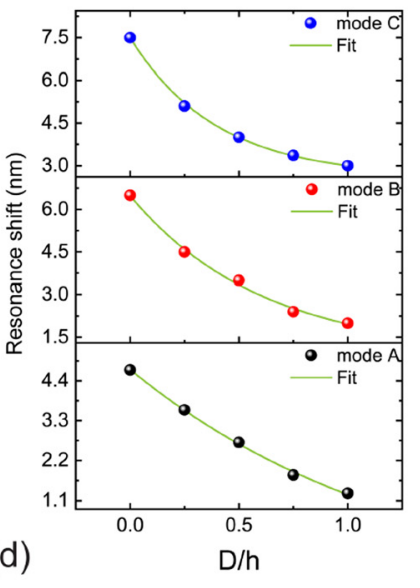

no binding

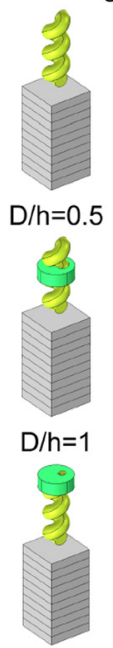

Figure 9: (a) Unit cell of the chiral metasurface hypergrating-hyperbolic metamaterials (CMH-HMM) simulated geometry. (b) Calculated TM-polarization reflectance spectra of an Au helix array on the HMM, with water as the surrounding medium, and angle of incidence $\theta_{i}=50^{\circ}$. The spectrum for pure water is shown in black, whereas red and blue curves correspond to two different mole fractions of 1,2,3-propantriol in distilled water. (c) Sketches of the simulated geometry with different percentages of the helix surfaces covered by bound analytes, and the corresponding resonance wavelength shift for the BPP modes as a function of the surface coverage. (d) Sketch of the geometry and resonance wavelength shift for mode $A, B$, and $C$ with surface coverage of $20 \%$, but with all analytes bound within a narrow disk at different distances $D$ away from the HMM surface. $D$ is normalized relative to the helix height $h$. is positioned at different distances $(D)$ with respect to the surface of the HMM. By plotting the resonance shift of the three modes A, B, and C as a function of the distance $D$ normalized to the helix height $(h)$ it is possible to see that the proposed biosensing platform is able to detect a shift of the considered modes even in the worst case $(D / h=1)$. For this case, the shift is about $1.3 \mathrm{~nm}$ for the mode $\mathrm{A}, 4.0 \mathrm{~nm}$ for the mode $B$, and $4.5 \mathrm{~nm}$ for the mode $C$, as reported in Figure 9d. These results highlight the advantages of having a metasurface to promote the detection of target analytes away from the surface of the HMM, exploiting the increased surface/volume ratio of the 3D CMH exposed to the analytes.

On the other hand, considering the intrinsic chirality of the nanohelices, $\mathrm{CMH}$ could excite new BPP modes of the HMM by coupling with their circular polarization-dependent plasmon modes. Indeed, as reported in Figure 10b, different reflectance dips are obtained for left-handed circular polarized (LCP) and right-handed circular polarized (RCP) light. In particular, the reflectance dips obtained for LCP light, indicated in the figure as $\mathrm{BPP}_{3}$ to $\mathrm{BPP}_{6}$, are strongly related to a coupling between the plasmonic modes of the gold helix array and the HMM as reported in Figure 10c.

From the chiroptical response of the $\mathrm{CMH}$, it is possible to calculate the CD signal, such as bipolar peaks and crossing points, as seen in Figure 10d. These signatures allow for increased sensitivity and accuracy when monitoring RI changes due to the analyte absorption. This sensing modality offers strong optical contrast even in the presence of highly achiral absorbing media, increasing the signal-to-noise CD measurements of a chiral analyte, relevant for complex biological media with limited transmission [123]. For this purpose, the reflectance curves obtained for LCP and RCP light at $\theta_{i}=75^{\circ}$ are used to calculate the reflectance circular dichroism (RCD) spectra, which characterizes the reflectance difference between LCP and RCP light, leading to an RCD amplitude $\left(\mathrm{RCD}=R_{\mathrm{LCP}}-R_{\mathrm{RCP}}\right)$. As expected, the RCD spectra for the CMH-HMM exhibit multiple features: different maxima, minima, and crossing points (Figure 10d) that are strongly affected by the RI variation (from 1.333 to 1.401). 
(a)
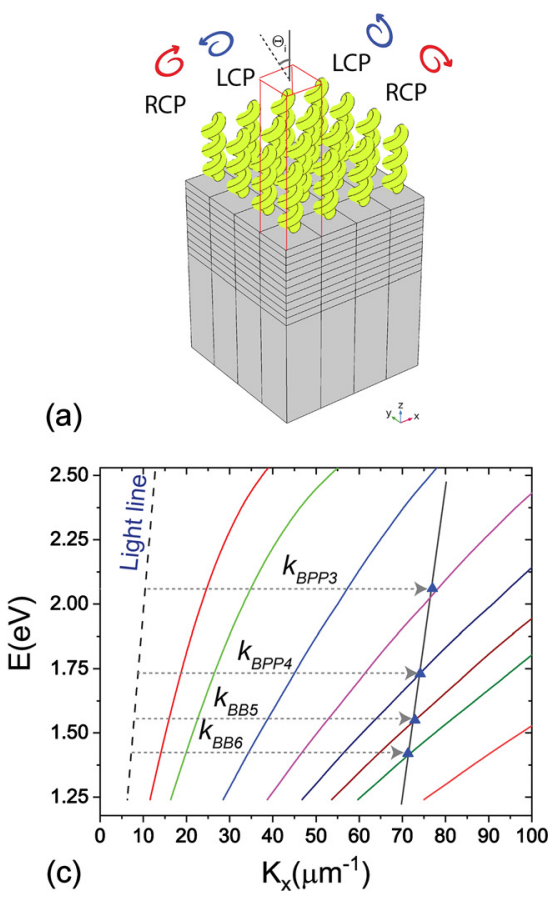
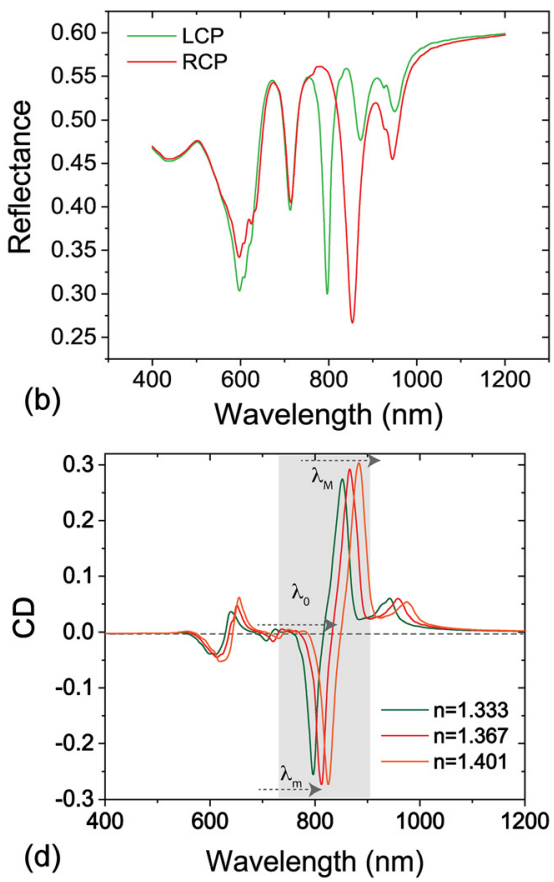

Figure 10: (a) Sketch of the simulated chiral metasurface hypergrating-hyperbolic metamaterials (CMH-HMM) unit cell probed with circular polarized light. (b) Reflectance curves of the CMH-HMM for left-handed circular polarized (LCP) and right-handed circular polarized (RCP) light, with angle of incidence $\theta_{i}=75^{\circ}$. (c) Modal dispersion curves of the HMM, with blue triangles indicating $\mathrm{BPP}$ modes $\mathrm{BPP}_{3}$ through $\mathrm{BPP}_{6}$ ) excited by circular polarized light. (d) Reflectance circular dichroism (RCD) versus wavelength at different refractive indices of the surrounding medium.
It is possible to distinguish in the range $700-900 \mathrm{~nm}$ a minimum $\left(\lambda_{m}\right)$, a crossing point $\left(\lambda_{0}\right)$ and a maximum $\left(\lambda_{M}\right)$, respectively, at 797,816 , and $852 \mathrm{~nm}$ which significantly shift and modify their intensity as the RI changes. These signals show a chiral plasmon (CP) sensitivity $S_{\mathrm{CP}}=\Delta \lambda / \Delta n$ of $412 \mathrm{~nm} / \mathrm{RIU}$ for $\lambda_{m}, 485 \mathrm{~nm} / \mathrm{RIU}$ for $\lambda_{0}$ and $471 \mathrm{~nm} / \mathrm{RIU}$ for $\lambda_{M}$, respectively. After extracting the classical full-width at half-maximum (FWHM) for the $\lambda_{m}$ and $\lambda_{M}$ modes and the FWHM in the $|R C D|$ spectrum for $\lambda_{0}$, the FOM, defined as $\mathrm{FOM}=S_{\mathrm{CP}} /$ FWHM [124], has been calculated. The corresponding FOM values are 18,20 , and 30 . The scientific significance of chiral metasurfaces coupled with HMM nanostructures for biosensing lies in the synergistic functionalities which arise from chiral geometries and optical selectivity. This will have important implication in developing next-generation biosensors for gene-protein recognition.

\section{Type I HMM-based biosensor}

\subsection{Type I HMMs: gold nanopillars for high- sensitivity LSPR sensors}

Kabashin et al. demonstrated an improvement in biosensing technology using a plasmonic metamaterial that is capable of supporting a guided mode in a porous nanorod layer [59]. Benefiting from a substantial overlap between the probing field and the active biological substance incorporated between the nanorods and a strong plasmonmediated energy confinement inside the layer, this type I metamaterial (Figure 11c) provides an enhanced sensitivity to RI variations of the medium between the rods (more than $30,000 \mathrm{~nm} / \mathrm{RIU}$ ). They focused on newly emerging plasmonic metamaterials, composite structures consisting of subwavelength-size components, and designed a sensororiented metamaterial that is capable of supporting similar or more sensitive guiding modes than SPPs. In particular, they reported on the experimental realization of such a metamaterial-based transducer using an array of parallel gold nanorods oriented normal to a glass substrate (Figure 11a and b). When the distance between the nanorods is smaller than the wavelength, this metamaterial layer supports a guided mode with the field distribution inside the layer determined by plasmon-mediated interaction between the nanorods. This anisotropic guided mode has resonant excitation conditions similar to the SPP mode of a smooth metal film and a large probe depth $(500 \mathrm{~nm})$. The structural parameters can be controlled by altering the fabrication conditions - typical ranges span rod lengths of $20-700 \mathrm{~nm}$, rod diameters $10-50 \mathrm{~nm}$, and separations $40-70 \mathrm{~nm}$ - thus achieving a nanorod areal density of approximately $10^{10}-10^{11} \mathrm{~cm}^{-2}$. The lateral size and separations between the nanorods are much smaller than the wavelength of light used in the experiments, so only average values of nanorod assembly parameters are important, and individual nanorod size deviations have no influence on the optical properties that are well described 
(a)
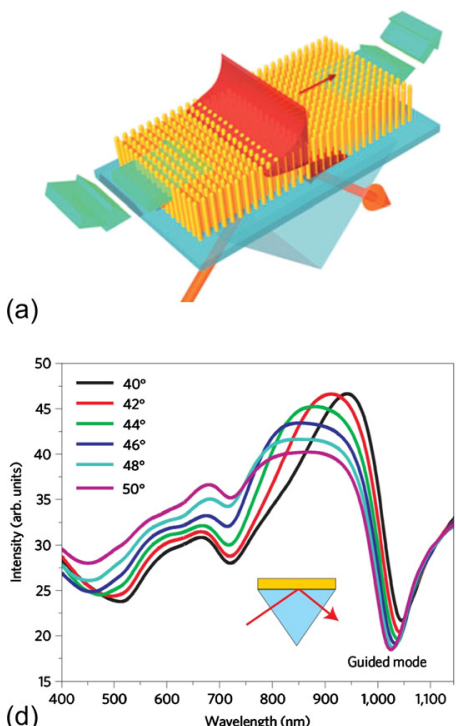

(b)
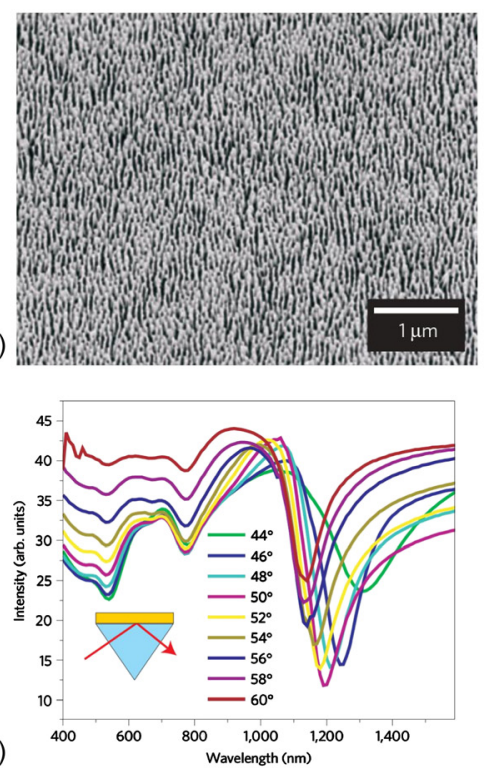
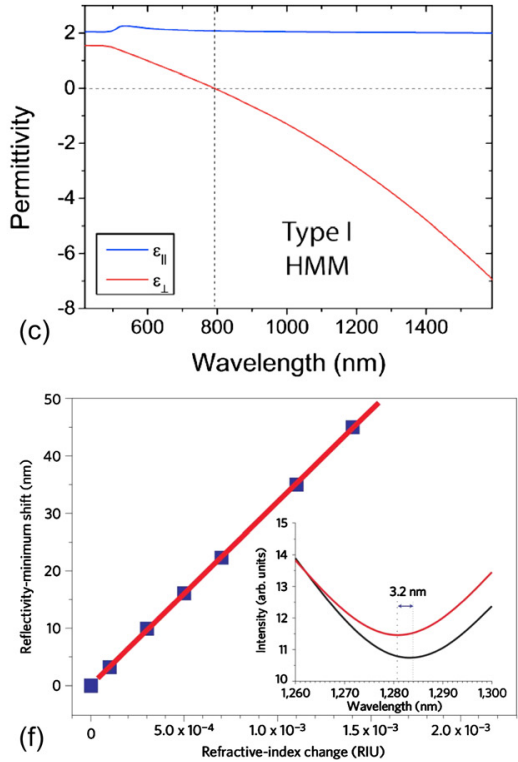

Figure 11: (a) Schematic of the gold nanopillars array and (b) scanning electron micrograph of the nanorod assembly. (c) Components of the effective permittivity tensor of the nanorod array in a water environment calculated using effective-medium theory components of the permittivity along the nanorods $\left(\varepsilon_{\|}\right)$and perpendicular to nanorods $\left(\varepsilon_{\perp}\right)$. (d) Reflection spectra of the nanorod array in an air ( $\left.n=1\right)$ (e) and water environment $(n=1.333)$, obtained in the attenuated total internal reflection (ATR) geometry for different angles of incidence. ( $f$ ) Calibration curve for the metamaterial-based sensor under the step-like changes of the refractive index of the environment using different glycerine-water solutions. The measurements were carried out at a wavelength of $1230 \mathrm{~nm}$. The size of the squares represents error bars. Inset: Reflectivity spectrum modifications with the changes of the refractive index by $10^{-4}$ RIU. Reproduced with permission [59]. Copyright 2009, Nature Publication Group.

by effective medium theory [125]. The nanorod structures' sensing properties were characterized in both direct transmission and attenuated total internal reflection (ATR) geometries using spectrometric and ellipsometric platforms.

In the direct-transmission geometry, the extinction spectra of the nanorod array in an air environment show two pronounced peaks at 520 and $720 \mathrm{~nm}$. These resonances correspond to the transverse and longitudinal modes of plasmonic excitations in the assembly [126-128]. The transverse mode is related to the electron motion perpendicular to the nanorod long axes and can be excited with light having an electric-field component perpendicular to it, whereas the longitudinal mode is related to the electron oscillations along the nanorod axes and requires $p$-polarized light to be excited with a component of the incident electric field along the nanorods. The electric-field distribution associated with the longitudinal mode has an intensity maximum in the middle of the nanorods and results from a strong dipole-dipole interaction between the plasmons of individual nanorods in the array and is, therefore, not sensitive to the presence of the superstrate [126-129]. Under oblique incidence and with $p$-polarized light, the electric field couples to both the transverse and longitudinal modes with the metamaterial slab showing extreme anisotropy of dielectric permittivity similar to that of a uniaxial crystal [130]. The illumination of the same nanorod structure in the ATR geometry reveals the new guided mode in the NIR spectral range which is excited only with $p$-polarized light and dominates the optical response of the assembly.

The metamaterial works similar to a conventional SPP-based sensor, showing a red shift of the resonance in response to an increase in the RI (Figure 11d and e). Furthermore, a change of the RI by $10^{-4}$ RIU causes a shift of the resonance by $3.2 \mathrm{~nm}$ even without any optimization of the structure (Figure 11f). The corresponding minimum estimation of sensitivity of $32,000 \mathrm{~nm} / \mathrm{RIU}$ exceeds the sensitivity of localized plasmon-based schemes by two orders of magnitude $[23,56,131,132]$ and an FOM value of 330 is achieved.

\subsection{Prism-coupled HMM-based tunable biosensor}

In this section, Sreekanth et al. demonstrated the development of a tunable type I HMM and excitation of BPP modes via prism coupling. By using the Goos-Hänchen 
(G-H) shift interrogation scheme, reconfigurable sensing with extreme sensitivity biosensing for small molecules has been shown [72]. The G-H shift describes the lateral displacement of the reflected beam from the interface of two media when the angles of incidence are close to the coupling angle [133].

As shown in Figure 12a, to develop a low-loss tunable HMM, 10 alternating thin layers of a low-loss plasmonic material such as TiN ( $16 \mathrm{~nm}$ ) and a low-loss phase change material such as $\mathrm{Sb}_{2} \mathrm{~S}_{3}(25 \mathrm{~nm})$ are deposited on a cleaned glass substrate. The optical properties of the developed HMM can be tuned by switching the structural phase of $\mathrm{Sb}_{2} \mathrm{~S}_{3}$ from amorphous to crystalline. In Figure 12b, the EMT-derived uniaxial permittivity components of $\mathrm{Sb}_{2} \mathrm{~S}_{3}-$ TiN HMM have been shown when $\mathrm{Sb}_{2} \mathrm{~S}_{3}$ is in amorphous and crystalline phases. As can be seen, $\mathrm{Sb}_{2} \mathrm{~S}_{3}-\mathrm{TiN} \mathrm{HMM}$ exhibits type I hyperbolic dispersion at $\lambda>580 \mathrm{~nm}$ when $\mathrm{Sb}_{2} \mathrm{~S}_{3}$ is in amorphous phase, where $\varepsilon_{\perp}=\varepsilon_{z z}<0$ and $\varepsilon_{\|}=\varepsilon_{x x}=\varepsilon_{y y}>0$. However, the operating wavelength of type I region is slightly blue shifted to $564 \mathrm{~nm}$ after the crystallization of $\mathrm{Sb}_{2} \mathrm{~S}_{33}$ layers in the HMM.

To excite the BPP modes of $\mathrm{Sb}_{2} \mathrm{~S}_{3}$-TiN HMM through prism coupling, a He-Ne laser $(632.8 \mathrm{~nm})$ with $p$-polarized light was used as the excitation source. This wavelength belongs to the hyperbolic region of the HMM and the effective index of HMM is less than that of the used BK7 prism index (1.5), so that the momentum matching condition can be satisfied. In Figure 12c, the excited BPP mode of $\mathrm{Sb}_{2} \mathrm{~S}_{3}$-TiN HMM have been plotted, when $\mathrm{Sb}_{2} \mathrm{~S}_{3}$ is in both phases. After switching the phase of $\mathrm{Sb}_{2} \mathrm{~S}_{3}$ from amorphous to crystalline state, the effective index of the HMM decreased, as a result (i) the minimum reflected intensity at the resonance angle declined, (ii) the linewidth of the reflection spectrum decreased, and (iii) the coupling angle slightly shifted. Figure $12 \mathrm{~d}$ shows the calculated dispersion diagram of fundamental BPP mode of the $\mathrm{Sb}_{2} \mathrm{~S}_{3}-\mathrm{TiN}$ HMM. Note that the experimentally determined parallel wavevector at $632.8 \mathrm{~nm}$ is exactly on the BPP dispersion curve of HMM. Then the Poynting vector $\left(S_{x}\right)$ of the HMM guided mode at $632.8 \mathrm{~nm}$ has been calculated (Figure 12e). It shows that the excited mode is a propagating wave inside the HMM, decays exponentially at HMM-water (air) interface, and is leaky in the prism. These are the basic characteristics of a typical BPP mode of an HMM. At resonance, the estimated propagating mode nearest in momentum is $13.08 \mu \mathrm{m}^{-1}$ with a propagation length of $177 \mathrm{~nm}$. It appears that the excited mode is the fundamental BPP mode of $\mathrm{Sb}_{2} \mathrm{~S}_{3}$-TiN HMM, but it is a low- $k$ mode. It is known that the phase difference between TM- and TE-polarized light experiences a sharp singularity at the coupling angle [134]. Thus, as shown in Figure 12f, this phase difference can be actively tuned by switching the phase of $\mathrm{Sb}_{2} \mathrm{~S}_{3}$ from amorphous to crystalline. (a)
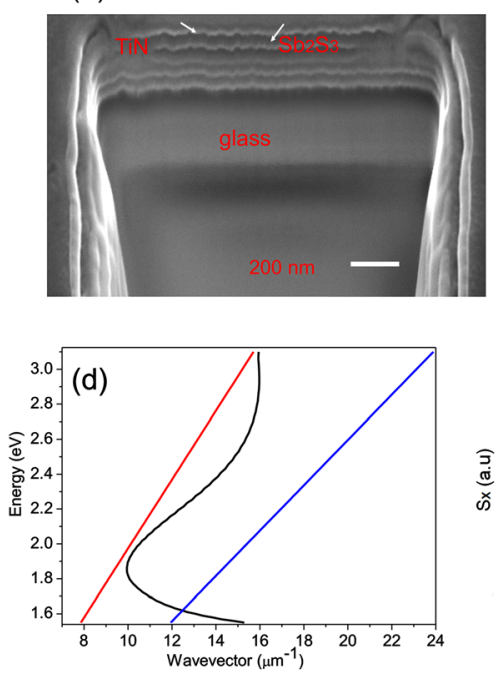

(b)

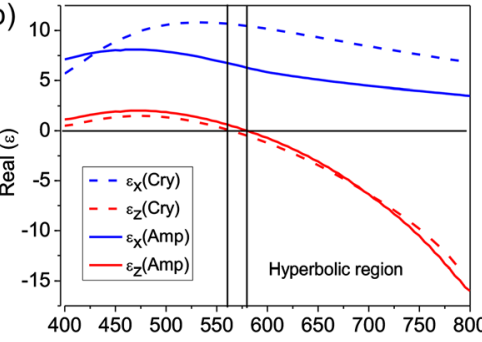

Wavelength $(\mathrm{nm})$

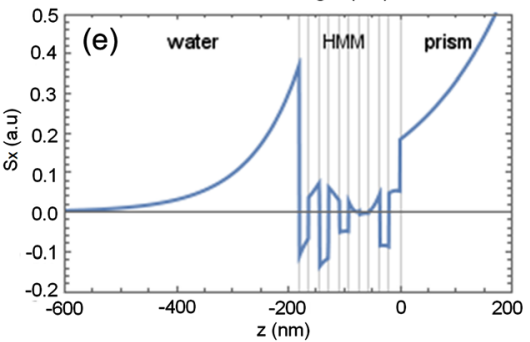

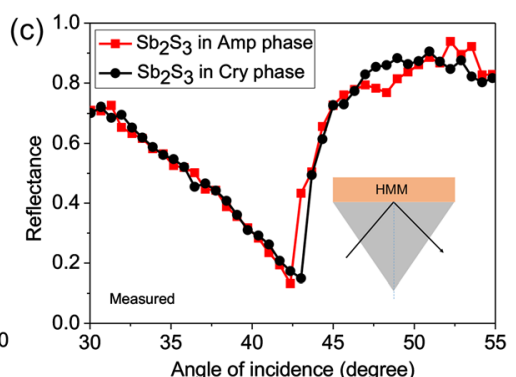

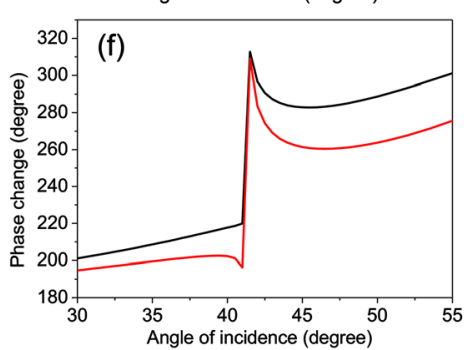

Figure 12: (a) An SEM image of a tunable $\mathrm{Sb}_{2} \mathrm{~S}_{3}-\mathrm{TiN} H M M$ consisting of five pairs of $\mathrm{Sb}_{2} \mathrm{~S}_{3}$ and TiN, with layer thicknesses of $25 \mathrm{~nm}$ for $\mathrm{Sb}_{2} \mathrm{~S}_{3}$ and $16 \mathrm{~nm}$ for TiN. (b) EMT-derived real parts of uniaxial permittivity components of $\mathrm{Sb}_{2} \mathrm{~S}_{3}-\mathrm{TiN} \mathrm{HMM}$ when $\mathrm{Sb}_{2} \mathrm{~S}_{3}$ is in the amorphous and crystalline phases. (c) Excited BPP mode of $\mathrm{Sb}_{2} \mathrm{~S}_{3}-\mathrm{TiN} \mathrm{HMM}$ for both phases of $\mathrm{Sb}_{2} \mathrm{~S}_{3}$. (d) Calculated dispersion diagram of fundamental BPP mode of $\mathrm{Sb}_{2} \mathrm{~S}_{3}-\mathrm{TiN}$ HMM. (e) Poynting vector of the guided mode of HMM. (f) Calculated phase difference between TM and TE polarization for both phases of $\mathrm{Sb}_{2} \mathrm{~S}_{3}$; Reproduced with permission from Sreekanth et al. [72]. Copyright 2019, John Wiley and Sons. 
Because the phase derivative at the coupling angle determines the magnitude of the $\mathrm{G}-\mathrm{H}$ shift, an enhanced and tunable $\mathrm{G}-\mathrm{H}$ shift at the BPP mode excitation angle can be realized using the $\mathrm{Sb}_{2} \mathrm{~S}_{3}$-TiN HMM. As shown in Figure 13a, the maximum $\mathrm{G}-\mathrm{H}$ shift is obtained at the coupling angle, where there is a sharp change of phase difference. In addition, a tunable $\mathrm{G}-\mathrm{H}$ shift is possible by switching the phase of $\mathrm{Sb}_{2} \mathrm{~S}_{3}$ in the HMM from amorphous to crystalline. To validate this tunable behavior experimentally, RI sensing has been performed because the $\mathrm{G}-\mathrm{H}$ shift strongly depends on the superstrate RI. A differential phase-sensitive setup to record the $\mathrm{GH}$ shifts has been used [133-135]. In the experiments, the G-H shifts have been monitored, by injecting different weight ratios $(1-10 \% \mathrm{w} / \mathrm{v})$ of aqueous solutions of glycerol with known refractive indices into sensor channel. The real-time RI sensing data are presented in Figure 13b, where measured G-H shift change with time due to the RI change of glycerol solutions has been report. It is evident that a clear step function in $\mathrm{G}-\mathrm{H}$ shift by varying the glycerol concentration is obtained. More importantly, a tunable G-H shift is obtained by switching the $\mathrm{Sb}_{2} \mathrm{~S}_{3}$ structural phase from amorphous to crystalline. It is clear from both calculations and experimental data that the maximum $\mathrm{G}-\mathrm{H}$ shift is possible when $\mathrm{Sb}_{2} \mathrm{~S}_{3}$ in the HMM is in the crystalline phase because the crystalline phase provides a higher phase change at the coupling angle. Therefore, the obtained maximum RI sensitivity for the crystalline phase of HMM results to be
$13.4 \times 10^{-7} \mathrm{RIU} / \mathrm{nm}$. The minimum sensitivity obtained for the amorphous phase of HMM is $16.3 \times 10^{-7} \mathrm{RIU} / \mathrm{nm}$. Because the sensor shows tunable sensitivity, a reconfigurable sensor device can be developed for future intelligent sensing applications. Although the obtained tunable range is small, the $\mathrm{G}-\mathrm{H}$ shift tunability can be further improved using longer wavelength sources and higher RI prisms.

Because enhanced RI sensitivity is possible with the $\mathrm{G}$ $\mathrm{H}$ shift interrogation scheme, it can be used to detect small biomolecules at extremely low concentrations. To demonstrate this, it was first sputtered a 10-nm film of gold on top of the crystalline HMM sample. Then the streptavidin-biotin affinity model protocol has been followed for the capture of biotin and the RI change caused by the capture of biotin on the sensor surface was recorded by measuring the $\mathrm{G}-\mathrm{H}$ shift. By injecting different concentrations (10 fM to $1 \mu \mathrm{M}$ ) of biotin prepared in PBS into the sensor channel with a sample volume of $98 \mu \mathrm{l}$ and the corresponding $\mathrm{G}-\mathrm{H}$ shift with increasing concentration was recorded. For biotin concentrations from $10 \mathrm{fM}$ to $1 \mu \mathrm{M}$, the response of the sensor with time was analyzed by calculating the marginal G-H shifts $\left(\delta \mathrm{GH} \mid \mathrm{GH}_{\mathrm{PBS}}-\mathrm{GH}_{\text {biotin }}\right)$. Figure $13 \mathrm{c}$ shows the recorded marginal G-H shifts after a reaction time of $40 \mathrm{~min}$ and an increase in marginal G-H shifts with increasing biotin concentration is obtained. To confirm the specific binding of biotin on streptavidin sites, the marginal $\mathrm{G}-\mathrm{H}$ shift change of a single measurement as the biotin molecules accumulate on the sensor surface over time has been recorded, as shown
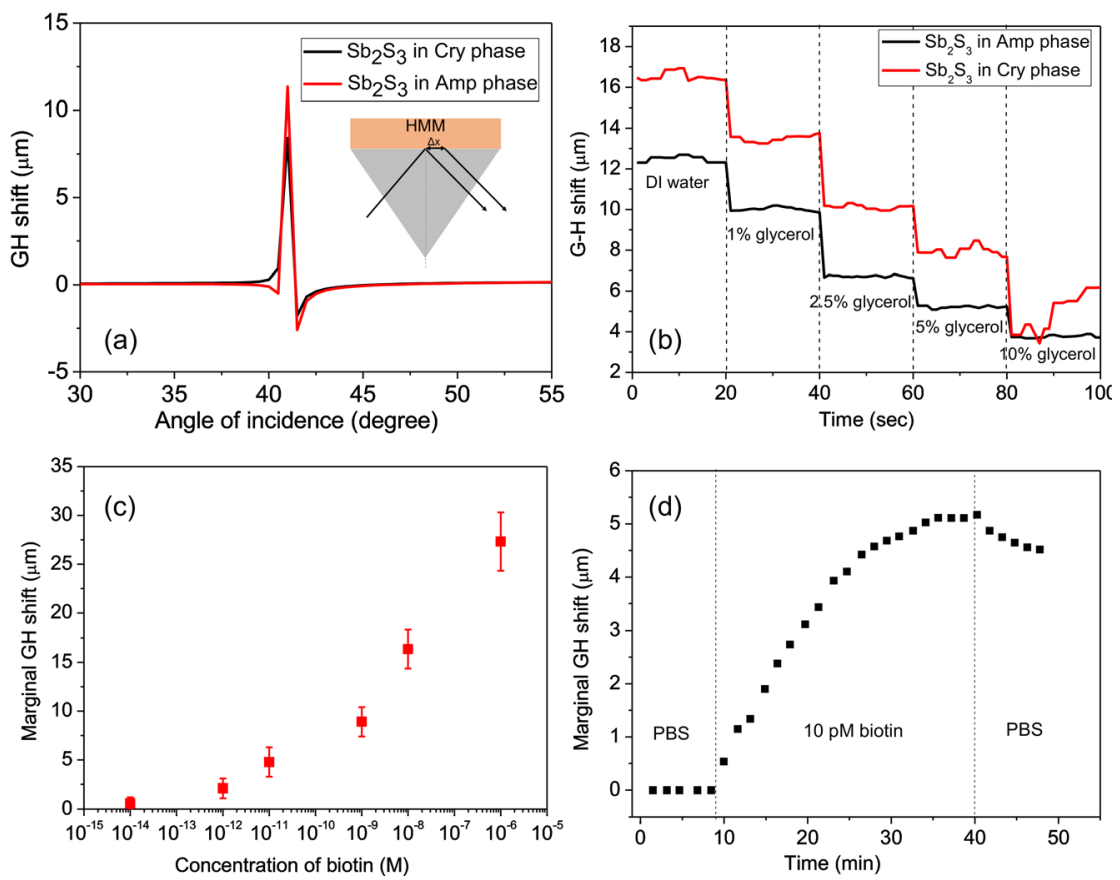

Figure 13: Demonstration of reconfigurable sensing using the Goos-Hänchen $(\mathrm{G}-\mathrm{H})$ shift interrogation scheme. (a) Calculated tunable GH shift of the HMM. (b) Real-time tunable refractive index sensing by injecting different weight percentage concentrations of glycerol in distilled water. (c, d) Demonstration of small molecule detection at low concentrations. (c) Measured marginal GH shift for different concentrations of biotin in PBS (10 fM to $1 \mu \mathrm{M}$ ) and (d) Variation of marginal GH shift over time with 10 pM biotin. Reproduced with permission from Sreekanth et al. [72]. Copyright 2019, John Wiley and Sons. 
in Figure 13d. A clear step in $\mathrm{G}-\mathrm{H}$ shift due to the binding of biotin molecules to streptavidin sites is observed. By considering the experimental noise level, it was possible to quantify that the detection limit of the sensor device is less than $1 \mathrm{pM}$. The detection of ultralow-molecular-weight biomolecules such as biotin at a low concentration of $1 \mathrm{pM}$ is occurred due to the extreme RI sensitivity of the sensor, which is achieved through the $\mathrm{G}-\mathrm{H}$ shift interrogation scheme. To conclude, it is possible to envision that small molecules such as exosomes can be detected even from bodily fluids using the proposed HMM-based plasmonic platform.

\section{Conclusions}

In this review, it has been reported the state of the art of hyperbolic dispersion metasurfaces and metamaterials and their applications in the field of molecular biosensing. We started by highlighting the physical aspects characterizing hyperbolic dispersion materials, followed by a review of some relevant designs and properties of HMM that have been proposed by different groups worldwide. HMMs have demonstrated to support extremely sensitive optical modes that can be used to develop cost-effective, noninvasive liquid biopsies for POC clinical evaluation, early cancer screening and real-time diagnosis of diseases. This review highlights the recent advances in hyperbolic dispersion metasurface-based biosensor technology, and in particular discusses biosensors involving metal/dielectric multilayered HMMs. This includes biosensors using different coupling mechanisms and interrogation schemes. We also discuss reconfigurable sensors based on tunable HMMs, LSPR sensors based on type I HMMs and biosensing applications of multifunctional hyperbolic nanocavities. The diagnostic platforms discussed here would call for radical changes in clinical diagnosis and treatment decisions, with the outcomes of lower capital costs, higher accuracy, and specificity. The development of optical devices based on radically new metasurfaces holds the promise to deliver an unprecedented detection limit (areal mass sensitivity at a level of $\mathrm{fg} / \mathrm{mm}$ ) with an optimal dynamic range for proteomic-genomic tests.

Acknowledgments: G.P. acknowledges support from the "AIM: Attraction and International Mobility" - PON R\&I 2014-2020 Calabria.

Author contribution: All the authors have accepted responsibility for the entire content of this submitted manuscript and approved submission.
Research funding: N.M. acknowledges support from the Luxembourg National Research Fund (CORE Grant No. C19/MS/13624497 "ULTRON") and from the FEDER Program (Grant No. 2017-03-022-19 “Lux-Ultra-Fast”). Conflict of interest statement: The authors declare no conflicts of interest regarding this article.

\section{References}

[1] W. T. Chen, A. Y. Zhu, and F. Capasso, "Flat optics with dispersion-engineered metasurfaces," Nat. Rev. Mater., vol. 5, pp. 604-620, 2020.

[2] N. Yu, P. Genevet, M. A. Kats, et al., "Light propagation with phase discontinuities: generalized laws of reflection and refraction," Science, vol. 334, no. 6054, pp. 333-337, 2011.

[3] M. Khorasaninejad and F. Capasso, "Metalenses: versatile multifunctional photonic components," Science, vol. 358, no. 6367, pp. 1-8, 2017.

[4] F. Capasso, "The future and promise of flat optics: a personal perspective," Nanophotonics, vol. 7, no. 6, pp. 953-957, 2018.

[5] N. Yu and F. Capasso, "Flat optics with designer metasurfaces," Nat. Mater., vol. 13, no. 2, pp. 139-150, 2014.

[6] S. M. Kamali, E. Arbabi, A. Arbabi, Y. Horie, and A. Faraon, "Highly tunable elastic dielectric metasurface lenses," Laser Photonics Rev., vol. 10, no. 6, pp. 1002-1008, 2016.

[7] E. Arbabi, A. Arbabi, S. M. Kamali, Y. Horie, M. Faraji-Dana, and A. Faraon, "Mems-tunable dielectric metasurface lens," Nat. Commun., vol. 9, no. 1, pp. 1-9, 2018.

[8] F. Ding, A. Pors, and S. I. Bozhevolnyi, “Gradient metasurfaces: a review of fundamentals and applications," Rep. Prog. Phys., vol. 81, no. 2, p. 026401, 2017.

[9] A. Tittl, A. John-Herpin, A. Leitis, E. R. Arvelo, and H. Altug, "Metasurface-based molecular biosensing aided by artificial intelligence," Angew. Chem. Int. Ed., vol. 58, no. 42, pp. 14810-14822, 2019.

[10] H. Altug, F. Yesilkoy, X. Li, et al., "Photonic metasurfaces for nextgeneration biosensors," in Integrated Photonics Research, Silicon and Nanophotonics, Optical Society of America, 2018, p. ITh3J-5.

[11] S. M. Choudhury, D. Wang, K. Chaudhuri, et al., "Material platforms for optical metasurfaces," Nanophotonics, vol. 7, no. 6, pp. 959-987, 2018.

[12] A. V. Kildishev, A. Boltasseva, and V. M. Shalaev, "Planar photonics with metasurfaces," Science, vol. 339, no. 6125, p. 1232009, 2013.

[13] S. M. Kamali, E. Arbabi, A. Arbabi, and A. Faraon, "A review of dielectric optical metasurfaces for wavefront control," Nanophotonics, vol. 7, no. 6, pp. 1041-1068, 2018.

[14] S. M. Kamali, E. Arbabi, A. Arbabi, Y. Horie, M. Faraji-Dana, and A. Faraon, “Angle-multiplexed metasurfaces: encoding independent wavefronts in a single metasurface under different illumination angles," Phys. Rev. X, vol. 7, no. 4, p. 041056 , 2017.

[15] S. M. Kamali, E. Arbabi, H. Kwon, and A. Faraon, "Metasurfacegenerated complex 3-dimensional optical fields for interference lithography," Proc. Natl. Acad. Sci., vol. 116, no. 43, pp. 21379-21384, 2019. 
[16] A. Leitis, A. Heßler, S. Wahl, et al., “Huygens’ metasurfaces: alldielectric programmable huygens' metasurfaces (adv. Funct. Mater. 19/2020)," Adv. Funct. Mater., vol. 30, no. 19, p. 2070122, 2020.

[17] F. Yesilkoy, E. R. Arvelo, Y. Jahani, et al., “Ultrasensitive hyperspectral imaging and biodetection enabled by dielectric metasurfaces," Nat. Photonics, vol. 13, no. 6, pp. 390-396, 2019.

[18] A. Tittl, A. Leitis, M. Liu, et al., "Imaging-based molecular barcoding with pixelated dielectric metasurfaces," Science, vol. 360, no. 6393, pp. 1105-1109, 2018.

[19] T. D. Gupta, L. Martin-Monier, W. Yan, et al., "Self-assembly of nanostructured glass metasurfaces via templated fluid instabilities," Nat. Nanotechnol., vol. 14, no. 4, pp. 320-327, 2019.

[20] H. Kwon, E. Arbabi, S. M. Kamali, M. Faraji-Dana, and A. Faraon, "Computational complex optical field imaging using a designed metasurface diffuser," Optica, vol. 5, no. 8, pp. 924-931, 2018.

[21] A. M. Shaltout, J. Kim, A. Boltasseva, V. M. Shalaev, and A. V. Kildishev, "Ultrathin and multicolour optical cavities with embedded metasurfaces," Nat. Commun., vol. 9, no. 1, pp. 1-7, 2018.

[22] J. Kim, S. Choudhury, C. DeVault, et al., "Controlling the polarization state of light with plasmonic metal oxide metasurface," ACS Nano, vol. 10, no. 10, pp. 9326-9333, 2016.

[23] B. Sepúlveda, P. C. Angelomé, L. M. Lechuga, and L. M. LizMarzán, "LSPR-based nanobiosensors," Nano Today, vol. 4, no. 3, pp. 244-251, 2009.

[24] A. Boltasseva and H. A. Atwater, "Low-loss plasmonic metamaterials,” Science, vol. 331, no. 6015, pp. 290-291, 2011.

[25] M. C. Estevez, M. Alvarez, and L. M. Lechuga, "Integrated optical devices for lab-on-a-chip biosensing applications," Laser Photonics Rev., vol. 6, no. 4, pp. 463-487, 2012.

[26] M.-C. Estevez, M. A. Otte, B. Sepulveda, and L. M. Lechuga, "Trends and challenges of refractometric nanoplasmonic biosensors: a review," Anal. Chim. Acta, vol. 806, pp. 55-73, 2014.

[27] X. Chen, L. Huang, H. Mühlenbernd, et al., "Dual-polarity plasmonic metalens for visible light," Nat. Commun., vol. 3, no. 1, pp. 1-6, 2012.

[28] K. A. Willets, A. J. Wilson, V. Sundaresan, and P. B. Joshi, "Superresolution imaging and plasmonics," Chem. Rev., vol. 117, no. 11, pp. 7538-7582, 2017.

[29] X. Huang, P. K. Jain, I. H. El-Sayed, and M. A. El-Sayed, "Plasmonic photothermal therapy (pptt) using gold nanoparticles," Laser Med. Sci., vol. 23, no. 3, p. 217, 2008.

[30] B. Nasseri, M. Turk, K. Kosemehmetoglu, et al., "The pimpled gold nanosphere: a superior candidate for plasmonic photothermal therapy," Int. J. Nanomed., vol. 15, p. 2903, 2020.

[31] L. Ricciardi, L. Sancey, G. Palermo, et al., "Plasmon-mediated cancer phototherapy: the combined effect of thermal and photodynamic processes," Nanoscale, vol. 9, no. 48, p. 19279-19289, 2017.

[32] M. R. Ali, M. A. Rahman, Y. Wu, et al., "Efficacy, long-term toxicity, and mechanistic studies of gold nanorods photothermal therapy of cancer in xenograft mice," Proc. Natl. Acad. Sci. U.S.A., vol. 114, no. 15, pp. E3110-E3118, 2017.

[33] M. Khan and H. Idriss, "Advances in plasmon-enhanced upconversion luminescence phenomena and their possible effect on light harvesting for energy applications," Wiley Interdiscip. Rev. Energy Environ., vol. 6, no. 6, p. e254, 2017.

[34] S. Manchala, L. R. Nagappagari, S. M. Venkatakrishnan, and V. Shanker, "Solar-light harvesting bimetallic $\mathrm{Ag} / \mathrm{Au}$ decorated graphene plasmonic system with efficient photoelectrochemical performance for the enhanced water reduction process," ACS Appl. Nano Mater., vol. 2, no. 8, pp. 4782-4792, 2019.

[35] M. Lee, J. U. Kim, J. S. Lee, B. I. Lee, J. Shin, and C. B. Park, "Mussel-inspired plasmonic nanohybrids for light harvesting," Adv. Mater., vol. 26, no. 26, pp. 4463-4468, 2014.

[36] M. Poudineh, E. H. Sargent, K. Pantel, and S. O. Kelley, “Profiling circulating tumour cells and other biomarkers of invasive cancers," Nat. Biomed. Eng., vol. 2, no. 2, pp. 72-84, 2018.

[37] V. Plaks, C. D. Koopman, and Z. Werb, "Circulating tumor cells," Science, vol. 341, no. 6151, pp. 1186-1188, 2013.

[38] M. G. Krebs, R. L. Metcalf, L. Carter, G. Brady, F. H. Blackhall, and C. Dive, "Molecular analysis of circulating tumour cells-biology and biomarkers," Nat. Rev. Clin. Oncol., vol. 11, no. 3, p. 129, 2014.

[39] M. Cristofanilli, G. T. Budd, M. J. Ellis, et al., "Circulating tumor cells, disease progression, and survival in metastatic breast cancer,” N. Engl. J. Med., vol. 351, no. 8, pp. 781-791, 2004.

[40] S. Maheswaran and D. A. Haber, "Circulating tumor cells: a window into cancer biology and metastasis," Curr. Opin. Genet. Dev., vol. 20, no. 1, pp. 96-99, 2010.

[41] S. Alimirzaie, M. Bagherzadeh, and M. R. Akbari, "Liquid biopsy in breast cancer: a comprehensive review," Clin. Genet., vol. 95, no. 6, pp. 643-660, 2019.

[42] C. Alix-Panabières and K. Pantel, "Circulating tumor cells: liquid biopsy of cancer," Clin. Chem., vol. 59, no. 1, pp. 110-118, 2013.

[43] E. I. Galanzha, Y. A. Menyaev, A. C. Yadem, et al., "In vivo liquid biopsy using cytophone platform for photoacoustic detection of circulating tumor cells in patients with melanoma," Sci. Transl. Med., vol. 11, no. 496, p. eaat5857, 2019.

[44] G. Rossi and M. Ignatiadis, "Promises and pitfalls of using liquid biopsy for precision medicine,” Canc. Res., vol. 79, no. 11, pp. 2798-2804, 2019.

[45] K. Boriachek, M. N. Islam, A. Möller, et al., "Biological functions and current advances in isolation and detection strategies for exosome nanovesicles," Small, vol. 14, no. 6, p. 1702153, 2018.

[46] T. Huang and C.-X. Deng, "Current progresses of exosomes as cancer diagnostic and prognostic biomarkers," Int. J. Biol. Sci., vol. 15, no. 1, p. 1, 2019.

[47] J.-H. Kim, E. Kim, and M. Y. Lee, "Exosomes as diagnostic biomarkers in cancer," Mol. Cell. Toxicol., vol. 14, no. 2, pp. 113-122, 2018.

[48] Y. H. Soung, T. Nguyen, H. Cao, J. Lee, and J. Chung, "Emerging roles of exosomes in cancer invasion and metastasis," $B M B$ Rep., vol. 49, no. 1, p. 18, 2016.

[49] I. Ament, J. Prasad, A. Henkel, S. Schmachtel, and C. Sonnichsen, "Single unlabeled protein detection on individual plasmonic nanoparticles," Nano Lett., vol. 12, no. 2, pp. 1092-1095, 2012.

[50] H. Im, H. Shao, Y. I. Park, et al., "Label-free detection and molecular profiling of exosomes with a nano-plasmonic sensor," Nat. Biotechnol., vol. 32, no. 5, pp. 490-495, 2014.

[51] A. A. I. Sina, R. Vaidyanathan, A. Wuethrich, L. G. Carrascosa, and M. Trau, "Label-free detection of exosomes using a surface plasmon resonance biosensor," Anal. Bioanal. Chem., vol. 411, no. 7, pp. 1311-1318, 2019. 
[52] S. S. Acimovic, M. A. Ortega, V. Sanz, et al., "Lspr chip for parallel, rapid, and sensitive detection of cancer markers in serum," Nano Lett., vol. 14, no. 5, pp. 2636-2641, 2014.

[53] V. Kravets, F. Schedin, R. Jalil, et al., "Singular phase nano-optics in plasmonic metamaterials for label-free single-molecule detection," Nat. Mater., vol. 12, no. 4, pp. 304-309, 2013.

[54] F. De Angelis, F. Gentile, F. Mecarini, et al., "Breaking the diffusion limit with super-hydrophobic delivery of molecules to plasmonic nanofocusing sers structures," Nat. Photonics, vol. 5, no. 11, pp. 682-687, 2011.

[55] P. Zijlstra, P. M. Paulo, and M. Orrit, "Optical detection of single non-absorbing molecules using the surface plasmon resonance of a gold nanorod," Nat. Nanotechnol., vol. 7, no. 6, pp. 379-382, 2012.

[56] J. N. Anker, W. P. Hall, O. Lyandres, N. C. Shah, J. Zhao, and R. P. Van Duyne, "Biosensing with plasmonic nanosensors," in Nanoscience and Technology: A Collection of Reviews from Nature Journals, Singapore, World Scientific, 2010, pp. 308-319.

[57] K. Q. Le, Q. M. Ngo, and T. K. Nguyen, “Nanostructured metalinsulator-metal metamaterials for refractive index biosensing applications: design, fabrication, and characterization," IEEE J. Sel. Top. Quant. Electron., vol. 23, no. 2, pp. 388-393, 2016.

[58] M. Svedendahl, R. Verre, and M. Käll, "Refractometric biosensing based on optical phase flips in sparse and shortrange-ordered nanoplasmonic layers," Light Sci. Appl., vol. 3, no. 11, p. e220, 2014.

[59] A. Kabashin, P. Evans, S. Pastkovsky, et al., "Plasmonic nanorod metamaterials for biosensing," Nat. Mater., vol. 8, no. 11, pp. 867-871, 2009.

[60] K. V. Sreekanth, Y. Alapan, M. ElKabbash, et al., "Extreme sensitivity biosensing platform based on hyperbolic metamaterials," Nat. Mater., vol. 15, no. 6, pp. 621-627, 2016.

[61] L. Jiang, S. Zeng, Z. Xu, et al., "Multifunctional hyperbolic nanogroove metasurface for submolecular detection," Small, vol. 13, no. 30, p. $1700600,2017$.

[62] G. Strangi, K. Sreekanth, and M. Elkabbash, "Hyperbolic metamaterial-based ultrasensitive plasmonic biosensors for early-stage cancer detection," in Next Generation Point-of-Care Biomedical Sensors Technologies for Cancer Diagnosis, Berlin, Springer, 2017, pp. 155-172.

[63] A. Poddubny, I. Iorsh, P. Belov, and Y. Kivshar, "Hyperbolic metamaterials," Nat. Photonics, vol. 7, no. 12, pp. 948-957, 2013.

[64] L. Ferrari, C. Wu, D. Lepage, X. Zhang, and Z. Liu, "Hyperbolic metamaterials and their applications," Prog. Quant. Electron., vol. 40, pp. 1-40, 2015.

[65] I. I. Smolyaninov and V. N. Smolyaninova, "Hyperbolic metamaterials: novel physics and applications," Solid State Electron., vol. 136, pp. 102-112, 2017.

[66] Z. Jacob and E. E. Narimanov, "Optical hyperspace for plasmons: Dyakonov states in metamaterials," Appl. Phys. Lett., vol. 93, no. 22, p. 221109, 2008.

[67] P. Shekhar, J. Atkinson, and Z. Jacob, "Hyperbolic metamaterials: fundamentals and applications," Nano Convergence, vol. 1, no. 1, p. 14, 2014.

[68] C. Cortes, W. Newman, S. Molesky, and Z. Jacob, "Quantum nanophotonics using hyperbolic metamaterials," J. Optics, vol. 14, no. 6, p. 063001, 2012.
[69] I. Avrutsky, I. Salakhutdinov, J. Elser, and V. Podolskiy, "Highly confined optical modes in nanoscale metal-dielectric multilayers," Phys. Rev. B, vol. 75, no. 24, p. 241402, 2007.

[70] K. V. Sreekanth, A. De Luca, and G. Strangi, “Experimental demonstration of surface and bulk plasmon polaritons in hypergratings," Sci. Rep., vol. 3, p. 3291, 2013.

[71] A. G. Brolo, "Plasmonics for future biosensors," Nat. Photonics, vol. 6, no. 11, pp. 709-713, 2012.

[72] K. V. Sreekanth, Q. Ouyang, S. Sreejith, et al., "Phase-changematerial-based low-loss visible-frequency hyperbolic metamaterials for ultrasensitive label-free biosensing," Adv. Opt. Mater., vol. 7, no. 12, p. 1900081, 2019.

[73] K. V. Sreekanth, M. ElKabbash, Y. Alapan, et al., "Hyperbolic metamaterials-based plasmonic biosensor for fluid biopsy with single molecule sensitivity," EPJ Appl. Metamater., vol. 4, p. 1, 2017.

[74] K. V. Sreekanth, Y. Alapan, M. ElKabbash, et al., "Enhancing the angular sensitivity of plasmonic sensors using hyperbolic metamaterials," Adv. Opt. Mater., vol. 4, no. 11, pp. 1767-1772, 2016.

[75] K. V. Sreekanth, P. Mahalakshmi, S. Han, M. S. Mani Rajan, P. K. Choudhury, and R. Singh, "Brewster mode-enhanced sensing with hyperbolic metamaterial," Adv. Opt. Mater., vol. 7, no. 21, p. 1900680, 2019.

[76] G. Palermo, G. E. Lio, M. Esposito, et al., "Biomolecular sensing at the interface between chiral metasurfaces and hyperbolic metamaterials," ACS Appl. Mater. Interfaces, vol. 12, no. 27 , pp. 30181-30188, 2020

[77] F. Abbas and M. Faryad, "A highly sensitive multiplasmonic sensor using hyperbolic chiral sculptured thin films," J. Appl. Phys., vol. 122, no. 17, p. 173104, 2017.

[78] J. Wu, F. Wu, C. Xue, et al., "Wide-angle ultrasensitive biosensors based on edge states in heterostructures containing hyperbolic metamaterials," Opt. Express, vol. 27, no. 17, pp. 24835-24846, 2019.

[79] W. He, Y. Feng, Z.-D. Hu, et al., "Sensors with multifold nanorod metasurfaces array based on hyperbolic metamaterials," IEEE Sens. J., vol. 20, no. 4, pp. 1801-1806, 2019.

[80] Y. Yoshida, Y. Kashiwai, E. Murakami, S. Ishida, and N. Hashiguchi, "Development of the monitoring system for slope deformations with fiber Bragg grating arrays," in Smart Structures and Materials 2002: Smart Sensor Technology and Measurement Systems, vol. 4694, International Society for Optics and Photonics, 2002, pp. 296-303.

[81] M. Baqir, A. Farmani, T. Fatima, M. Raza, S. Shaukat, and A. Mir, "Nanoscale, tunable, and highly sensitive biosensor utilizing hyperbolic metamaterials in the near-infrared range," Appl. Opt., vol. 57, no. 31, pp. 9447-9454, 2018.

[82] N. Vasilantonakis, G. Wurtz, V. Podolskiy, and A. Zayats, "Refractive index sensing with hyperbolic metamaterials: strategies for biosensing and nonlinearity enhancement," Opt. Express, vol. 23, no. 11, pp. 14329-14343, 2015.

[83] D. Rodrigo, O. Limaj, D. Janner, et al., "Mid-infrared plasmonic biosensing with graphene," Science, vol. 349, no. 6244, pp. 165-168, 2015.

[84] G. E. Lio, A. Ferraro, M. Giocondo, R. Caputo, and A. De Luca, "Color Gamut behavior in epsilon near-zero nanocavities during propagation of gap surface plasmons," Adv. Opt. Mater., vol. 18, no. 17 , p. 2000487,2020 
[85] X. Yang, J. Yao, J. Rho, X. Yin, and X. Zhang, “Experimental realization of three-dimensional indefinite cavities at the nanoscale with anomalous scaling laws," Nat. Photonics, vol. 6, no. 7, pp. 450-454, 2012.

[86] C. Guclu, T. S. Luk, G. T. Wang, and F. Capolino, "Radiative emission enhancement using nano-antennas made of hyperbolic metamaterial resonators," Appl. Phys. Lett., vol. 105, no. 12, p. 123101, 2014.

[87] D. Lu, J. J. Kan, E. E. Fullerton, and Z. Liu, “Enhancing spontaneous emission rates of molecules using nanopatterned multilayer hyperbolic metamaterials," Nat. Nanotechnol., vol. 9, no. 1, pp. 48-53, 2014.

[88] D. Lu, H. Qian, K. Wang, et al., “Nanostructuring multilayer hyperbolic metamaterials for ultrafast and bright green ingan quantum wells," Adv. Mater., vol. 30, no. 15, p. 1706411, 2018.

[89] S. C. Indukuri, J. Bar-David, N. Mazurski, and U. Levy, "Ultrasmall mode volume hyperbolic nanocavities for enhanced light-matter interaction at the nanoscale," ACS Nano, vol. 13, no. 10, pp. 11770-11780, 2019.

[90] P. R. West, N. Kinsey, M. Ferrera, A. V. Kildishev, V. M. Shalaev, and A. Boltasseva, "Adiabatically tapered hyperbolic metamaterials for dispersion control of high- $k$ waves," Nano Lett., vol. 15, no. 1, pp. 498-505, 2015.

[91] T. Galfsky, H. Krishnamoorthy, W. Newman, E. Narimanov, Z. Jacob, and V. Menon, "Active hyperbolic metamaterials: enhanced spontaneous emission and light extraction," Optica, vol. 2, no. 1, pp. 62-65, 2015.

[92] N. Maccaferri, T. Isoniemi, M. Hinczewski, M. Iarossi, G. Strangi, and F. De Angelis, "Designer bloch plasmon polariton dispersion in grating-coupled hyperbolic metamaterials," APL Photonics, vol. 5, no. 7, p. 076109, 2020.

[93] F. Peragut, L. Cerutti, A. Baranov, et al., "Hyperbolic metamaterials and surface plasmon polaritons," Optica, vol. 4, no. 11, pp. 1409-1415, 2017.

[94] T. Isoniemi, N. Maccaferri, Q. M. Ramasse, G. Strangi, and F. De Angelis, "Electron energy loss spectroscopy of bright and dark modes in hyperbolic metamaterial nanostructures," Adv. Opt. Mater., vol. 8, no. 13, p. 2000277, 2020.

[95] N. Maccaferri, Y. Zhao, T. Isoniemi, et al., "Hyperbolic metaantennas enable full control of scattering and absorption of light," Nano Lett., vol. 19, no. 3, pp. 1851-1859, 2019.

[96] J. Song and W. Zhou, "Multiresonant composite optical nanoantennas by out-of-plane plasmonic engineering," Nano Lett., vol. 18, no. 7, pp. 4409-4416, 2018.

[97] P. Wang, A. V. Krasavin, F. N. Viscomi, et al., "Metaparticles: Dressing nano-objects with a hyperbolic coating," Laser Photonics Rev., vol. 12, no. 11, p. 1800179, 2018.

[98] J. Zhou, A. F. Kaplan, L. Chen, and L. J. Guo, "Experiment and theory of the broadband absorption by a tapered hyperbolic metamaterial array," ACS Photonics, vol. 1, no. 7, pp. 618-624, 2014.

[99] M. Sakhdari, M. Hajizadegan, M. Farhat, and P.-Y. Chen, "Efficient, broadband and wide-angle hot-electron transduction using metal-semiconductor hyperbolic metamaterials," Nano Energy, vol. 26, pp. 371-381, 2016.

[100] G. Abdelatif, M. F. O. Hameed, S. Obayya, and M. Hussein, "Ultrabroadband absorber based on a funnel-shaped anisotropic metamaterial," JOSA B, vol. 36 , no. 10 , pp. 2889-2895, 2019.
[101] C. T. Riley, J. S. Smalley, J. R. Brodie, Y. Fainman, D. J. Sirbuly, and Z. Liu, "Near-perfect broadband absorption from hyperbolic metamaterial nanoparticles," Proc. Natl. Acad. Sci. U.S.A., vol. 114, no. 6, pp. 1264-1268, 2017.

[102] V. Caligiuri, L. Pezzi, A. Veltri, and A. De Luca, "Resonant gain singularities in 1D and 3D metal/dielectric multilayered nanostructures," ACS Nano, vol. 11, no. 1, pp. 1012-1025, 2017.

[103] W. D. Newman, C. L. Cortes, J. Atkinson, S. Pramanik, R. G. DeCorby, and Z. Jacob, "Ferrell-Berreman modes in plasmonic epsilon-near-zero media," ACS Photonics, vol. 2, no. 1, pp. 2-7, 2015.

[104] R. A. Ferrell, "Predicted radiation of plasma oscillations in metal films," Phys. Rev., vol. 111, no. 5, p. 1214, 1958.

[105] C. Xu, J. Xu, G. Song, C. Zhu, Y. Yang, and G. S. Agarwal, "Enhanced displacements in reflected beams at hyperbolic metamaterials," Opt. Express, vol. 24, no. 19, pp. 21767-21776, 2016.

[106] G. Luka, A. Ahmadi, H. Najjaran, et al., “Microfluidics integrated biosensors: a leading technology towards lab-on-a-chip and sensing applications," Sensors, vol. 15, no. 12, pp. 30011-30031, 2015.

[107] K. Sreekanth, A. De Luca, and G. Strangi, “Excitation of volume plasmon polaritons in metal-dielectric metamaterials using 1D and 2D diffraction gratings," J. Opt., vol. 16, no. 10, p. 105103, 2014.

[108] K. V. Sreekanth, K. H. Krishna, A. De Luca, and G. Strangi, “Large spontaneous emission rate enhancement in grating coupled hyperbolic metamaterials," Sci. Rep., vol. 4, p. 6340, 2014.

[109] S. Thongrattanasiri and V. A. Podolskiy, "Hypergratings: nanophotonics in planar anisotropic metamaterials," Opt. Lett., vol. 34, no. 7, pp. 890-892, 2009.

[110] S. Zeng, D. Baillargeat, H.-P. Ho, and K.-T. Yong, "Nanomaterials enhanced surface plasmon resonance for biological and chemical sensing applications," Chem. Soc. Rev., vol. 43, no. 10, pp. 3426-3452, 2014.

[111] M. Esposito, V. Tasco, M. Cuscuna, et al., "Nanoscale 3D chiral plasmonic helices with circular dichroism at visible frequencies," ACS Photonics, vol. 2, no. 1, pp. 105-114, 2015.

[112] M. Esposito, V. Tasco, F. Todisco, et al., "Programmable extreme chirality in the visible by helix-shaped metamaterial platform," Nano Lett., vol. 16, no. 9, pp. 5823-5828, 2016.

[113] J. Rho, and X. Zhang, "Recent progress in hyperbolic, chiral metamaterials and metasurfaces," in Workshop on Optical Plasmonic Materials, Optical Society of America, 2014, p. OW4D-1.

[114] F. Li, T. Tang, J. Li, et al., "Chiral coding metasurfaces with integrated vanadium dioxide for thermo-optic modulation of terahertz waves," J. Alloys Compd., vol. 826, p. 154174, 2020.

[115] O. Kotov and Y. E. Lozovik, "Enhanced optical activity in hyperbolic metasurfaces," Phys. Rev. B, vol. 96, no. 23, p. 235403, 2017.

[116] Y. Zhao, A. N. Askarpour, L. Sun, J. Shi, X. Li, and A. Alù, "Chirality detection of enantiomers using twisted optical metamaterials," Nat. Commun., vol. 8, no. 1, pp. 1-8, 2017.

[117] Z. Wang, Y. Wang, G. Adamo, et al., "A novel chiral metasurface with controllable circular dichroism induced by coupling localized and propagating modes," Adv. Opt. Mater., vol. 4, no. 6, pp. 883-888, 2016.

[118] M. L. Solomon, J. Hu, M. Lawrence, A. García-Etxarri, and J. A. Dionne, "Enantiospecific optical enhancement of chiral 
sensing and separation with dielectric metasurfaces," ACS

Photonics, vol. 6, no. 1, pp. 43-49, 2018.

[119] E. Yashima, K. Maeda, and T. Nishimura, "Detection and amplification of chirality by helical polymers," Chem. Eur. J., vol. 10, no. 1, pp. 42-51, 2004.

[120] J. T. Collins, C. Kuppe, D. C. Hooper, C. Sibilia, M. Centini, and V. K. Valev, "Chirality and chiroptical effects in metal nanostructures: fundamentals and current trends," Adv. Opt. Mater., vol. 5, no. 16, p. 1700182, 2017.

[121] G. E. Lio, G. Palermo, R. Caputo, and A. De Luca, “A comprehensive optical analysis of nanoscale structures: from thin films to asymmetric nanocavities," RSC Adv., vol. 9, no. 37, pp. 21429-21437, 2019.

[122] F. Koohyar, F. Kiani, S. Sharifi, M. Sharifirad, and S. H. Rahmanpour, "Study on the change of refractive index on mixing, excess molar volume and viscosity deviation for aqueous solution of methanol, ethanol, ethylene glycol, 1-propanol and 1,2,3-propantriol at $t=292.15 \mathrm{~K}$ and atmospheric pressure," Res. J. Appl. Sci. Eng. Technol., vol. 4, no. 17, pp. 3095-3101, 2012.

[123] H.-H. Jeong, A. G. Mark, M. Alarcón-Correa, et al., "Dispersion and shape engineered plasmonic nanosensors," Nat. Commun., vol. 7, no. 1, pp. 1-7, 2016.

[124] K. M. Mayer and J. H. Hafner, "Localized surface plasmon resonance sensors,” Chem. Rev., vol. 111, no. 6, pp. 3828-3857, 2011.

[125] T. C. Choy, Effective Medium Theory: Principles and Applications, vol. 165, Oxford, Oxford University Press, 2015.

[126] P. R. Evans, G. A. Wurtz, R. Atkinson, et al., "Plasmonic core/ shell nanorod arrays: subattoliter controlled geometry and tunable optical properties," J. Phys. Chem. C, vol. 111, no. 34, pp. 12522-12527, 2007.
[127] G. Wurtz, W. Dickson, D. O'connor, et al., “Guided plasmonic modes in nanorod assemblies: strong electromagnetic coupling regime," Opt. Express, vol. 16, no. 10, pp. 7460-7470, 2008.

[128] W. Dickson, G. Wurtz, P. Evans, et al., “Dielectric-loaded plasmonic nanoantenna arrays: a metamaterial with tuneable optical properties," Phys. Rev. B, vol. 76, no. 11, p. 115411, 2007.

[129] G. A. Wurtz, P. R. Evans, W. Hendren, et al., "Molecular plasmonics with tunable exciton-plasmon coupling strength in j-aggregate hybridized au nanorod assemblies," Nano Lett., vol. 7, no. 5, pp. 1297-1303, 2007.

[130] J. Elser, R. Wangberg, V. A. Podolskiy, and E. E. Narimanov, "Nanowire metamaterials with extreme optical anisotropy," Appl. Phys. Lett., vol. 89, no. 26, p. 261102, 2006.

[131] B. Sepúlveda, A. Calle, L. M. Lechuga, and G. Armelles, “Highly sensitive detection of biomolecules with the magneto-optic surface-plasmon-resonance sensor," Opt. Lett., vol. 31, no. 8, pp. 1085-1087, 2006.

[132] M. E. Stewart, C. R. Anderton, L. B. Thompson, et al., "Nanostructured plasmonic sensors," Chem. Rev., vol. 108, no. 2, pp. 494-521, 2008.

[133] K. V. Sreekanth, Q. Ouyang, S. Han, K.-T. Yong, and R. Singh, "Giant enhancement in Goos-Hänchen shift at the singular phase of a nanophotonic cavity," Appl. Phys. Lett., vol. 112, no. 16, p. 161109, 2018.

[134] K. V. Sreekanth, S. Sreejith, S. Han, et al., "Biosensing with the singular phase of an ultrathin metal-dielectric nanophotonic cavity," Nat. Commun., vol. 9, no. 1, pp. 1-8, 2018.

[135] K. V. Sreekanth, W. Dong, Q. Ouyang, et al., "Large-area silverstibnite nanoporous plasmonic films for label-free biosensing," ACS Appl. Mater. Interfaces, vol. 10, no. 41, pp. 34991-34999, 2018. 